\title{
Conditioning Method Dramatically Alters the Role of Amygdala in Taste Aversion Learning
}

\author{
Glenn E. Schafe, ${ }^{1}$ Todd E. Thiele, and Ilene L. Bernstein \\ Department of Psychology \\ University of Washington \\ Seattle, Washington 98195 USA
}

\begin{abstract}
Although an important role for the amygdala in taste aversion learning has been suggested by work in a number of laboratories, results have been inconsistent and interpretations varied. The present series of studies reevaluated the role of the amygdala in taste aversion learning by examining the extent to which conditioning methods, testing methods and lesioning methods, influence whether amygdala lesions dramatically affect conditioned taste aversion (CTA) learning. Results indicated that when animals are conditioned with an intraoral (I/O) taste presentation, lesions of amygdala eliminate evidence of conditioning whether animals are tested intraorally or with a two-bottle solution presentation. Dramatic effects of amygdala lesions on CTA learning were seen whether lesions were made electrolytically or using an excitotoxin. In contrast, when animals were conditioned using bottle presentation of the taste, electrolytic lesions attenuated CTAs but did not eliminate them, and excitotoxic lesions had no effect. These results are consistent with the hypothesis that neural structures critical for CTA learning may differ depending on the extent to which the method of conditioned stimulus delivery incorporates a response component.
\end{abstract}

\section{Introduction}

Taste aversion learning is a robust form of as-

\footnotetext{
${ }^{1}$ Corresponding author.

Present address: Center for Neural Science, New York, New York 10003 USA.
}

sociative learning in which animals and humans learn to avoid a taste or flavor that has been followed by gastrointestinal malaise (Garcia et al. 1974; Bernstein 1991). In the laboratory, conditioned taste aversions (CTAs) are commonly established in a single trial by exposing animals to a taste conditioned stimulus (CS) followed by administration of a malaise-producing drug, such as $\mathrm{LiCl}$ [unconditioned stimulus (US)].

Taste aversion learning has received extensive experimental attention (e.g., see Riley and Clarke 1977). However, unlike other defensive conditioning paradigms, a clear definition of the neural circuitry underlying this unusual type of learning has yet to emerge (Chambers 1990; Yamamoto 1993). Recent studies have pointed to the importance of the pontine parabrachial nucleus (PBN) in acquisition but not expression of CTAs (Reilly et al. 1993; Grigson et al. 1997). However, because the chronic decerebrate rat is unable to acquire a CTA (Grill and Norgren 1978), forebrain structures appear to be necessary for CTA learning. Identification of those structures, however, has remained elusive. Studies examining the effects of amygdala lesions on CTA learning have been particularly inconsistent. Several laboratories report that lesions of the amygdala significantly interfere with CTA learning (Nachman and Ashe 1974; Lasiter and Glanzman 1985; Simbayi et al. 1986; Gallo et al. 1992; Kesner et al. 1992; Yamamoto et al. 1995), whereas others find little or no effect (BermudezRattoni and McGaugh 1991; Hatfield et al. 1992; Galaverna et al. 1993). Of those who do find effects, some point to the importance of the central nucleus (Lasiter and Glanzman 1985), whereas others have implicated the basolateral nucleus (Nachman and Ashe 1974; Simbayi et al. 1986; Yamamoto and Fugimoto 1991; Yamamoto 1993). Finally, a compelling case has been made that when amygdala lesions do interfere with CTA learning,

LEARNING \& MEMORY 5:481-492 @ 1998 by Cold Spring Harbor Laboratory Press ISSN1072-0502/98 \$5.00

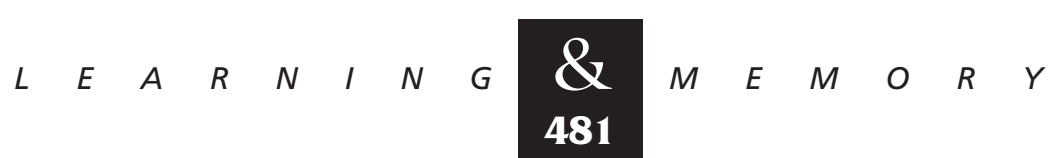




\section{Schafe et al.}

the effect is principally owing to damage to fibers passing from insular cortex through amygdala (Dunn and Everitt 1988).

In contrast to these variable results, our laboratory recently obtained clear and consistent effects of lesions of the amygdala on CTA learning (Schafe and Bernstein 1996). The striking result was the complete elimination of evidence of CTA conditioning in lesioned animals, an observation that contrasts with that of the majority of prior studies that have found attenuation, but not necessarily elimination, of CTA learning after amygdala lesions (Yamamoto and Fujimoto 1991; Gallo et al. 1992; Kesner et al. 1992). One key difference between our amygdala lesion studies and those of other laboratories was that our CTA studies used direct infusion of CS solutions into the oral cavity both during conditioning and testing (see Fig. 1, top), largely because of our interest in a striking cellular correlate of CTA expression, c-Fos induction in the intermediate division of the nucleus of the solitary tract (iNTS) (Swank and Bernstein 1994). This involuntary CS-delivery protocol contrasts with most CTA studies in the literature in which animals initiate CS exposure voluntarily by drinking solution from a bottle (see Fig. 1, bottom) and, as such, could be an important procedural factor in determining whether amygdala lesions significantly affect taste aversion learning.
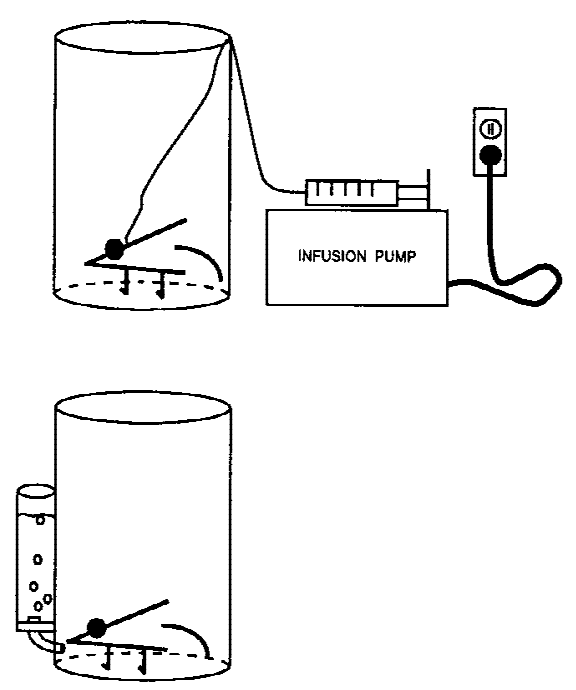

Figure 1: Schematic illustration of different methods used to establish a CTA. Rats are either infused intraorally with a CS taste solution followed by injection with toxic drug, such as $\mathrm{LiCl}$ (top), or are allowed to drink the CS taste solution from a bottle followed by the same drug (bottom).
These considerations led us to systematically examine whether conditioning, testing, and/or lesioning methods influence the effects of amygdala lesions on CTA learning. Given the variability in results of past studies evaluating effects of amygdala lesions on CTA, we chose to lesion the entire amygdala rather than evaluate the role of specific subnuclei. Results indicated that when animals were conditioned with an intraoral (I/O) CS presentation, lesions of amygdala eliminated evidence of conditioning whether animals were tested intraorally or with a two-bottle solution presentation. Elimination of aversions was found whether lesions were made electrolytically or using an excitotoxin. In contrast, when animals were conditioned using a more conventional bottle presentation of the CS, electrolytic lesions of amygdala attenuated CTAs but did not eliminate them, whereas excitotoxic lesions had no effect. Thus, the involvement of amygdala in CTA learning appears to depend on the extent to which the delivery of the CS in the conditioning protocol incorporates a response requirement.

\section{General Methods}

\section{SUBJECTS}

Adult male Long-Evans rats were obtained from the breeding colony maintained at the University of Washington. At the time of surgery, freefeeding body weights ranged from 300 to 400 grams. Rats were housed individually in suspended stainless steel cages and maintained on a 12:12-hr light/dark cycle. Teklad rodent chow and water were provided ad libitum unless otherwise indicated.

\section{LESIONS}

Under Equithesin $(3.3 \mathrm{mg} / \mathrm{kg})$ anesthesia, rats were first implanted with an I/O cannula and then given either bilateral lesions of the amygdala or SHAM operations. The oral cannula was constructed of 100-gauge polyethylene tubing and was inserted with a 19-gauge sharpened stainless steel probe. The probe was inserted through the roof of the mouth just anterolateral to the first maxillary molar and passed through the cheek, caudal to the eye, to exit the scapular area behind the head. Electrolytic lesions of the amygdala were produced by passage of $20 \mathrm{sec}$ of 2-mA anodal current through an exposed tip of a Teflon-insulated tungsten elec- 
trode (0.008 inch, A.M. Systems, Everett, WA). Lesion coordinates, taken from Paxinos and Watson (1986) and adjusted with pilot data, were 1.80$3.30 \mathrm{~mm}$ posterior to bregma, $4.4-4.7 \mathrm{~mm}$ lateral to the midline, and $8.2-8.8 \mathrm{~mm}$ ventral to the skull surface (for a total of four penetrations). For SHAM animals, the skull was opened and the dura penetrated, but no lesion was made. Excitotoxic lesions of the amygdala were produced using ibotenic acid. A single penetration was made on each side of the brain, at the following coordinates: 2.50 $\mathrm{mm}$ posterior to bregma, $4.4 \mathrm{~mm}$ lateral to the midline, and $8.1 \mathrm{~mm}$ ventral to the skull surface. A 23-gauge guide cannula was attached to the manipulator arm of the stereotaxic device. Ibotenic acid (RBI; $10 \mathrm{mg} / \mathrm{ml}, 0.5 \mu \mathrm{l}$ ) was dissolved in sterile PBS and infused slowly $(0.1 \mu \mathrm{l} / \mathrm{min})$ via infusion pump through a 30-gauge injector cannula. Following infusion, the injector cannula was left in place for an additional $5 \mathrm{~min}$ to allow diffusion of the toxin from the cannula tip. At the time of surgery, rats received $0.2 \mathrm{ml}$ of Gentamicin sulfate ( $40 \mathrm{mg}$ / $\mathrm{ml}$, i.m.) as a prophylaxis against infection. Weights were taken every other day, and animals were given at least 7 days to recover prior to habituation and conditioning.

\section{HISTOLOGY}

To verify the location and completeness of the lesions, 50- $\mu \mathrm{m}$ sections were cut in the transverse plane throughout a $3-\mathrm{mm}$ region circumscribing the amygdala. Every other section was mounted onto gelatin-coated glass slides. Sections were air dried, stained for Nissl using Cresyl violet, dehydrated in ethanol, cleared in Histoclear, and coverslipped using Permount. Camera lucida drawings of sections were prepared and examined using a projection light microscope. Sections were airdried, stained for Nissl using Cresyl violet, dehydrated in ethanol, cleared in Histoclear, and coverslipped using Permount. Camera lucida drawings of sections were prepared and examined using a projection light microscope. Sections were analyzed by comparing them with those found in Swanson (1994).

\section{Experiment 1: Evaluation of Testing} Method on the Effects of Electrolytic Amygdala Lesions on CTA Learning

In the first experiment, rats with bilateral electrolytic lesions of amygdala were conditioned us- ing an I/O solution presentation (Fig. 1, top). Previous studies in our laboratory have shown complete elimination of CTA learning in amygdalalesioned rats conditioned with this method (Schafe and Bernstein 1996). Those studies did not, however, evaluate whether the elimination of CTA learning following I/O conditioning generalizes to other types of testing situations. In this experiment, we therefore used both $\mathrm{I} / \mathrm{O}$ tests and a more conventional two-bottle test to evaluate this question.

\section{Materials and Methods}

\section{HABITUATION AND CONDITIONING}

In the 5 days following cannula implantation and electrolytic lesions, the oral cannula was flushed every other day with distilled water to prevent clogging. Two days prior to conditioning, rats were habituated to Plexiglas cylindrical chambers that were to be used during conditioning. On each habituation day, rats were placed in a cylinder for $30 \mathrm{~min}$ and infused intraorally by infusion pump with $5 \mathrm{ml}$ of distilled water at a rate of $0.5 \mathrm{ml} / \mathrm{min}$. Rats in each surgical group (SHAM and Lesion) were assigned to experimental groups: SHAMPaired $(n=5)$, SHAM-Unpaired $(n=5)$, LesionPaired $(n=7)$, and Lesion-Unpaired $(n=5)$.

On the conditioning day, experimental ("paired") animals recived a single conditioning trial within the chamber consisting of I/O exposure to $5 \mathrm{ml}$ of $0.15 \%$ saccharin at a rate of 0.5 $\mathrm{ml} / \mathrm{min}$ followed immediately by injection of 0.15 M LiCl (20 ml/kg, i.p.). Control ("unpaired") animals were infused with saccharin followed by injection of an equivalent volume of $0.15 \mathrm{~m} \mathrm{NaCl}$. Rats were videotaped during infusions to later assess the latency to which any rejection responses (described below) occurred. On the day following conditioning, paired animals received noncontingent injections of $0.15 \mathrm{M} \mathrm{NaCl}$, whereas unpaired animals received noncontingent injections of 0.15 M LiCl. This was done to ensure equivalent exposure to LiCl (US) and saccharin (CS), with the only difference between groups being whether the two stimuli were paired.

\section{TESTING}

Two days following conditioning, rats were subjected to two types of tests to assess CTAs: latency to reject I/O infusion of the taste CS and the 
more commonly used two-bottle choice test with water. For the I/O test, rats were placed in the chamber and reinfused intraorally with the CS taste ( $5 \mathrm{ml}, 0.5 \mathrm{ml} / \mathrm{min}$ ). As during conditioning, animals were videotaped for subsequent analysis of rejection responses. Following saccharin infusion, rats were returned to their home cage. For the two-bottle test, rats were given a 12-hr two-bottle preference test between $0.15 \%$ saccharin and water. The order of I/O and two-bottle tests was counterbalanced such that half the rats in each group received the $\mathrm{I} / \mathrm{O}$ test first and half received the two-bottle test first.

\section{QUANTIFICATION OF BEHAVIOR}

Videotapes of animals exposed to the CS taste during the I/O test were viewed and scored by an observer blind to experimental condition. For each rat, latency to reject the saccharin was recorded. As in our previous studies, rejection latency was defined in terms of the time elapsing between onset of the I/O infusion and the onset of persistent, passive dripping of the solution from the oral cavity. Mann-Whitney U-tests were used to evaluate differences between groups. For the two-bottle test, solution intake was measured to the nearest gram. Data were analyzed with analysis of variance (ANOVA) and post-hoc $t$-tests.

\section{Results and Discussion}

\section{I/O TEST}

Mean latency to reject the I/O infusion of CS saccharin following conditioning is presented in Figure 2. Consistent with our previous findings (Schafe and Bernstein 1996), paired rats with lesions of amygdala showed virtually no evidence of aversion conditioning, ingesting the saccharin throughout most of the $10-\mathrm{min}$ infusion period and appearing indistinguishable from unpaired controls. Mann-Whitney $U$-tests determined that the effects of both saccharin-drug pairing and lesion on time to reject were significant (Sham-Paired vs. Sham-Unpaired, $P<0.01$; Sham-Paired vs. LesionPaired, $P<0.01$; Sham-Paired vs. Lesion-Unpaired, $P<0.01)$. No differences were detected between paired and unpaired lesioned animals or Sham-Unpaired animals and the lesioned groups. Thus, when conditioned and tested intraorally, rats with bilateral electrolytic lesions of amygdala show no evidence of aversion conditioning.

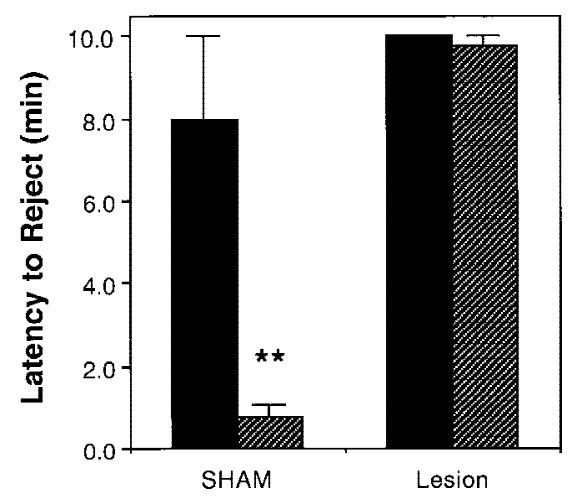

Figure 2: Mean rejection latency ( \pm S.E. $)$ of SHAM and electrolytically lesioned animals during I/O saccharin infusion at the time of testing. Saccharin had either been paired (hatched bars) or unpaired (solid bars) with $\mathrm{LiCl}$ using $\mathrm{I} / \mathrm{O}$ conditioning. $\left.{ }^{* *}\right) P<0.01$ relative to unpaired controls (experiment 1).

\section{TWO-BOTTLE TEST}

Preference ratios and absolute solution intake of both the saccharin CS and water are presented in Figure 3 (top and bottom, respectively). Consistent with the results of the I/O test, paired rats with lesions of amygdala showed virtually no evidence of aversion conditioning, displaying a clear preference for the saccharin CS over the water and appearing indistinguishable from unpaired controls. Results of the ANOVA revealed a significant main effect for saccharin-drug pairing, $[F(1,18)=14.67, P<0.01]$, a significant effect for lesion, $[F(1,18)=12.03, P<0.01]$, and a significant drug pairing by lesion interaction $[F(1,18)=6.06, P<0.05]$. These results are also reflected in the absolute solution intakes, in which intake of the saccharin in both lesioned groups (paired and unpaired) was statistically indistinguishable from that of unpaired animals. Thus, when conditioned intraorally, rats with electrolytic lesions of amygdala show no evidence of aversion conditioning in either an involuntary I/O test or a voluntary two-bottle preference test, indicating that the effect of amygdala lesions was independent of the type of test used to assess conditioning.

\section{Experiment 2: Evaluation of Conditioning Method on the Effects of Electrolytic Amygdala Lesions on CTA Learning}

In the previous experiment, we showed that electrolytic lesions of amygdala eliminate evidence

$$
\begin{array}{lllllllllllllll}
L & E & A & R & N & I & N & G & \underset{\mathbf{4 8 4}}{\mathbf{Q}} & M & E & M & O & R & Y
\end{array}
$$



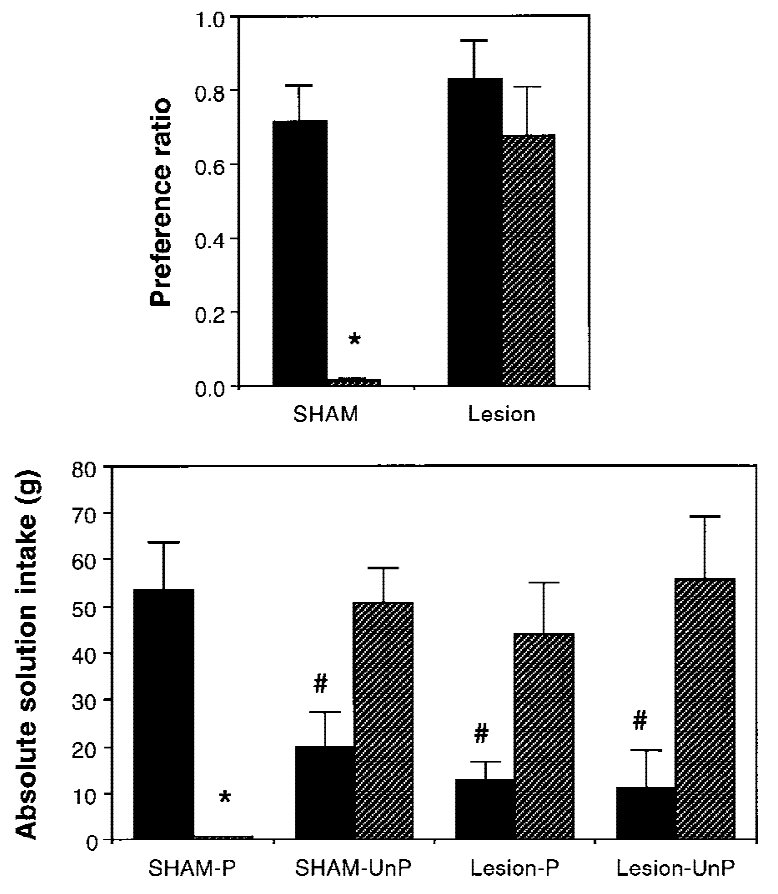

Figure 3: (top) Mean ( \pm S.E.) preference ratios for SHAm and electrolytically lesioned animals following 12-hr two-bottle test between saccharin and water. Saccharin had either been paired (hatched bars) or unpaired (solid bars) with $\mathrm{LiCl}$ using $\mathrm{I} / \mathrm{O}$ conditioning. (Bottom) Mean ( \pm S.E.) absolute solution intake of water (solid bars) and saccharin (hatched bars) during the same test. $\left(^{*}\right)$ $P<0.01$ relative to unpaired controls; (\#) $P<0.01$ relative to water intake in SHAM-Paired controls (experiment 1).

of CTA learning in rats conditioned with the I/O method, regardless of whether they are tested intraorally or with a more conventional two-bottle test. In this experiment, a more conventional conditioning method was used; namely, rats with comparable electrolytic lesions of amygdala were exposed during conditioning to the CS taste by drinking the solution from a bottle (Fig. 1, bottom).

\section{Materials and Methods}

\section{HABITUATION AND CONDITIONING}

One week following the placement of electrolytic lesions, rats were acclimated to a water deprivation schedule consisting of $1.5 \mathrm{hr}$ of access to water per day, divided into two drinking periods. One-half hour of water access was followed by an additional hour of hydration several hours later. Prior to conditioning, rats were given 3 days of habituation to drinking in the cylindrical chambers by allowing them their initial half hour of water access from a drinking tube attached to the chamber wall. Prior to conditioning, rats in each surgical group (SHAM and Lesion) were assigned to experimental groups: SHAM-Paired $(n=5)$, SHAM-Unpaired $(n=4)$, Lesion-Paired $(n=5)$, and LesionUnpaired $(n=4)$.

On the conditioning day, paired animals received a single conditioning trial within the chamber consisting of half an hour of access to a bottle of $0.15 \%$ saccharin followed immediately by injection of $0.15 \mathrm{~m} \mathrm{LiCl}(20 \mathrm{ml} / \mathrm{kg}$, i.p.). Unpaired animals were given access to the saccharin followed by injection of an equivalent volume of $0.15 \mathrm{M}$ $\mathrm{NaCl}$. On the day following conditioning, rats received noncontingent injections as in experiment 1. All rats were returned to ad libitum access to water and given an additional week to recover from conditioning and deprivation prior to testing.

\section{TESTING}

On the test day, rats were given a 12-hr twobottle preference test between $0.15 \%$ saccharin solution and water as described in Experiment 1.

\section{Results and Discussion}

Preference ratios and absolute solution intake of both the saccharin CS and water are presented in Figure 4 (top and bottom, respectively). Unlike the results of the previous experiment, paired lesioned animals showed evidence of aversion conditioning, although aversions were weaker than those of unlesioned animals. Results of the ANOVA on saccharin preference ratios revealed a significant main effect for saccharin-drug pairing $[F(1,14)=24.46, P<0.01]$ and a significant effect for lesion $[F(1,14)=8.67, P<0.01]$. The interaction, however, was not found to be significant.

At first glance, the pattern of results for saccharin preference ratios in Figures 3 (I/O conditioning) and 4 (bottle conditioning) appears similar except that the paired versus unpaired lesion groups achieve significance in the second experiment. However, the difference between the first and second experiment emerges more clearly when absolute intakes are examined. Here, it can be seen that in the first experiment the pattern of saccharin intake of Lesion-Paired and Lesion-Unpaired animals is remarkably similar; both groups drink much more saccharin than water (Fig. 3, bot-

$$
\begin{array}{lllllllllllllll}
\hline & E & A & R & N & I & N & G & \begin{array}{r}
\boldsymbol{Q} \\
485
\end{array} & M & E & M & O & R & Y
\end{array}
$$



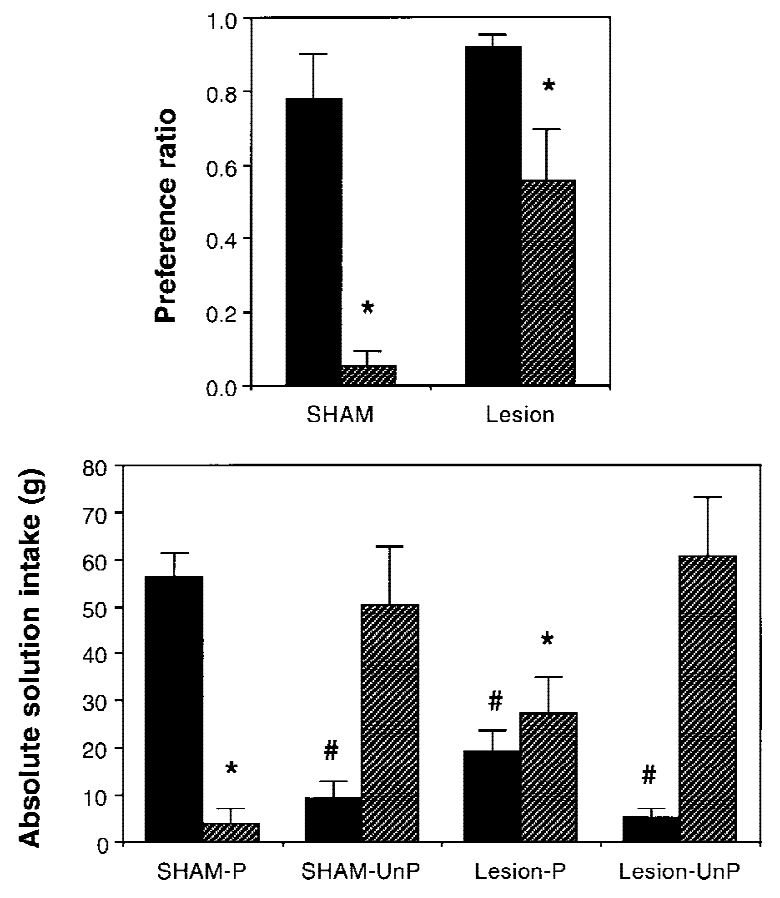

Figure 4: (Top) Mean ( \pm S.E.) preference ratios for SHAM and electrolytically lesioned animals following 12-hr two-bottle test between sacharin and water. Saccharin had either been paired (hatched bars) or unpaired (solid bars) with $\mathrm{LiCl}$ using bottle conditioning. (Bottom) Mean ( \pm S.E.) absolute solution intake of water (solid bars) and saccharin (hatched bars) during the same test. $\left(^{*}\right)$ $P<0.01$ relative to unpaired controls; (\#) $P<0.01$ relative to water intake in SHAM-Paired controls (experiment 2).

tom). This is contrasted with intakes of animals in the second experiment where Lesion-Unpaired animals markedly prefer saccharin to water, whereas Lesion-Paired animals drink about equal amounts of the two fluids (Fig. 4, bottom). Statistically, this was confirmed; Lesion-Paired animals consumed less saccharin than unpaired controls $(P<0.05)$, although their intake was still higher than that of SHAM-Paired animals. Thus, unlike results when animals were trained using the $\mathrm{I} / \mathrm{O}$ method, rats with electrolytic amygdala lesions that were trained using a bottle showed evidence of CTA learning; there was attenuation but not elimination of the learning.

Because Figures 3 and 4 display the same dependent measures after conditioning with two different methods, they allow us to assess whether the strength of conditioning with bottle and I/O presentations are comparable. A comparison of Sham-Paired animals in the two studies provides evidence that the tendency to avoid the LiCl-paired saccharin is comparable despite the use of two different conditioning methods. Thus, differences in the effects of amygdala lesions on CTA learning do not appear to be attributable to differences in the strength of the learning.

\section{HISTOLOGY}

Reconstructions of the rostrocaudal extent of electrolytic amygdala lesions in experiments 1 (I/O conditioning) and 2 (bottle conditioning) are presented on the left and right sides of Figure 5, respectively. Examination of the sections revealed that lesioned animals sustained damage to a variety of subnuclei of the amygdala, including the basolateral, lateral, and central amygdaloid nuclei. In most animals, some damage to the ventral aspect of the globus pallidus and striatum was also noted. A few animals had damage to the lateral aspect of the internal capsule. A careful comparison of lesions in the first and second experiments indicates that the lesions were comparable. If anything, the lesions in the second study were somewhat more extensive. Thus, the observed differences in degree of interference with CTA learning in the two studies cannot be attributed to incomplete or smaller lesions in experiment 2 .

\section{Experiment 3: Evaluation of Lesioning Method on the Effects of Amygdala Lesions on CTA Learning}

The previous two experiments used electrolytic lesions of amygdala. Consistent with previous findings, electrolytic lesions eliminated evidence of CTA learning in rats conditioned using the I/O method (Schafe and Bernstein 1996) and attenuated, but did not eliminate, evidence of conditioning in bottle-trained animals (Yamamoto and Fujimoto 1991; Gallo et al. 1992; Kesner et al. 1992). To determine whether one or both of these effects might be attributable to damage to fibers passing through amygdala (Dunn and Everitt 1988), the following set of experiments used excitotoxic lesions, which spare fibers of passage (Jarrard 1991). As before, conditioning methods involved both $\mathrm{I} / \mathrm{O}$ solution presentation (experiment $3 \mathrm{a}$ ) and bottle solution presentation (experiment $3 \mathrm{~b}$ ).

\section{Materials and Methods}

\section{HABITUATION AND CONDITIONING}

For experiment $3 \mathrm{a}$, rats with $\mathrm{I} / \mathrm{O}$ cannulas and bilateral ibotenic acid lesions of amygdala were ha-

$$
\begin{array}{lllllllllllllll}
L & E & A & R & N & I & N & G & \underset{486}{\mathbf{Z}} & M & E & M & O & R & Y
\end{array}
$$


I/O Conditioned

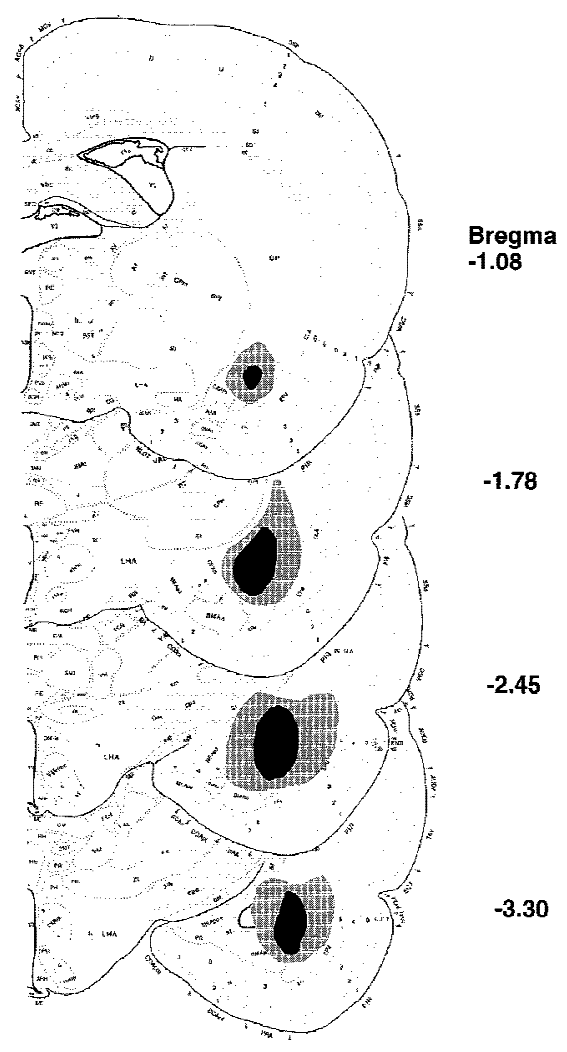

Bottle Conditioned

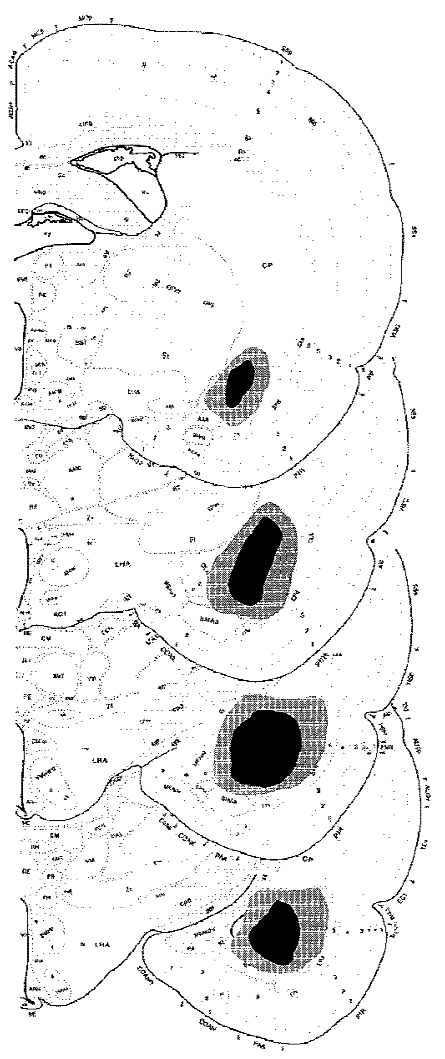

Figure 5: Serial reconstructions of electrolytic amygdala lesions in the transverse plane. (Left) The extent of the lesions in experiment 1 , in which animals were conditioned intraorally. (Right) The extent of the lesions in experiment 2 , in which rats were conditioned with a bottle. The lightly and darkly shaded regions correspond to the largest and smallest lesions, respectively. bituated to chambers and I/O infusions as described previously and conditioned and tested using the $\mathrm{I} / \mathrm{O}$ method of experiment 1 . There were three groups in this experiment: SHAM-Paired $(n=3)$, Lesion-Paired $(n=10)$, and Lesion-Unpaired $(n=3)$. For experiment $3 \mathrm{~b}$, separate groups of similarly lesioned animals were conditioned using the bottle presentation methods of Experiment 2 . There were also three groups in this experiment: SHAM-Paired $(n=5)$, Lesion-Paired $(n=7)$, and Lesion-Unpaired $(n=5)$.

\section{TESTING}

As in the previous experiments, on the test day rats were tested either by assessing the latency to reject I/O infusion of the taste CS (experiment 3a) or by a 12-hr two-bottle preference test between the CS solution and water (experiment $3 \mathrm{~b}$ ).

\section{Results and Discussion}

\section{EXPERIMENT 3a}

Mean latency to reject the I/O infusion of CS saccharin during testing is presented in Figure 6. It can be seen that excitotoxic lesions had essentially the same effect on CTA expression as electrolytic lesions; namely, lesioned animals showed little or no evidence of CTA learning. Aversions were evident in SHAM-Paired animals but not in LesionPaired animals; Lesion-Paired animals were significantly different from the SHAM-Paired $(P<0.05)$

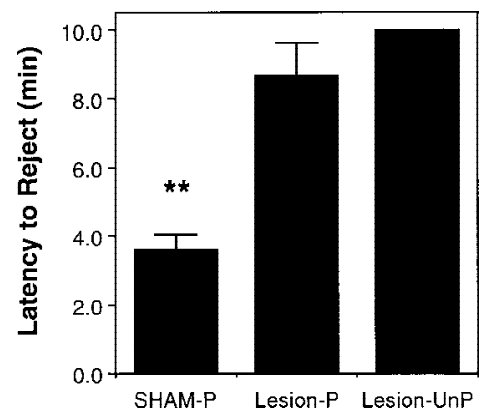

Figure 6: Mean rejection latency $( \pm$ S.E. $)$ of SHAM and excitotoxically lesioned animals during I/O saccharin infusion at the time of testing. Saccharin had either been paired $(\mathrm{P})$ or unpaired (UnP) with $\mathrm{LiCl}$ using $\mathrm{I} / \mathrm{O}$ conditioning. $\left.{ }^{*}\right) P<0.01$ relative to lesioned groups (experiment 3a).

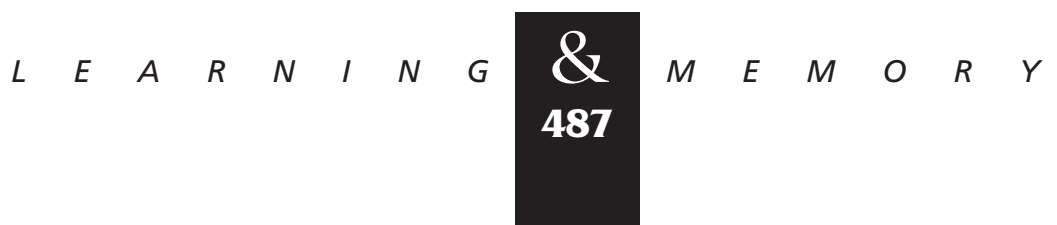




\section{Schafe et al.}

but not the lesion-unpaired group. In fact, 8 of 10 animals in the Lesion-Paired group continued to ingest the saccharin throughout the 10-min infusion period, showing no signs of passive dripping.

\section{EXPERIMENT 3b}

Preference ratios and absolute solution intake of both the saccharin CS and water are presented in Figure 7 (top and bottom, respectively). Unlike the results of the previous experiment, paired lesioned animals showed strong aversion conditioning (Lesion-Paired vs. Lesion-Unpaired, $P<0.01$; Duncan's test). Results of experiment $3 \mathrm{~b}$ are in striking contrast with those of experiment $3 \mathrm{a}$; in $3 \mathrm{a}$ no evidence of aversion conditioning is seen in lesioned animals, whereas in $3 \mathrm{~b}$ aversion conditioning appears normal. The lesioning methods were identical, as were the CS and US. The most
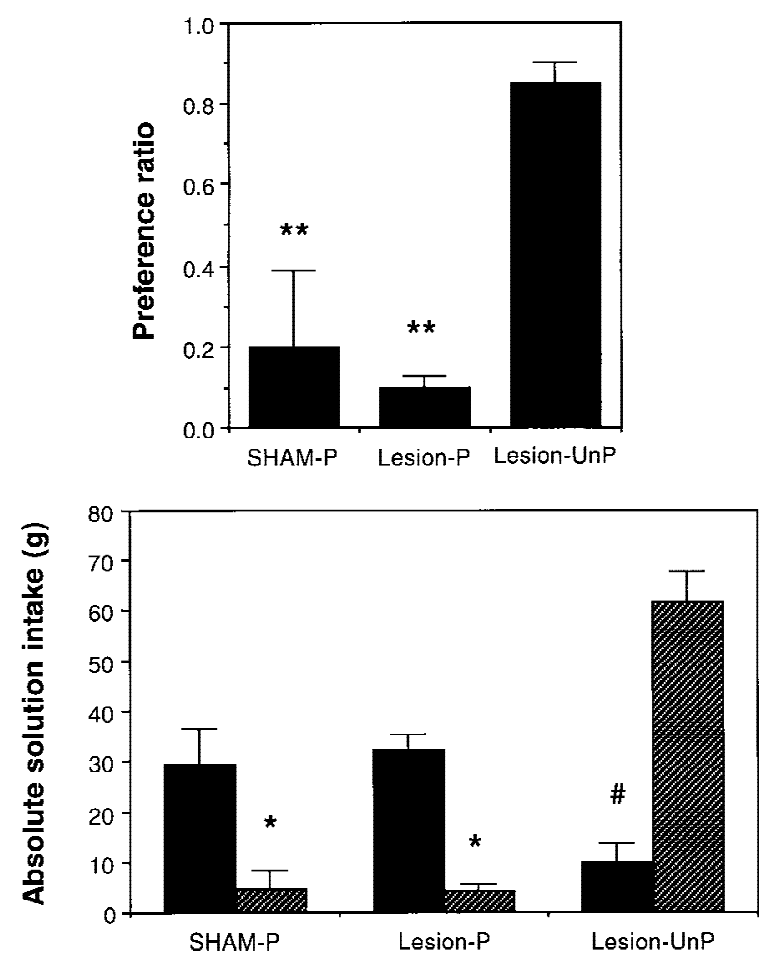

Figure 7: (Top) Mean ( \pm S.E.) preference ratios for SHAM and excitotoxically lesioned animals following 12-hr two-bottle test between saccharin and water. Saccharin had either been paired $(\mathrm{P})$ or unpaired $(\mathrm{UnP})$ with $\mathrm{LiCl}$ using bottle conditioning. (Bottom) Mean ( \pm S.E.) absolute solution intake of water (solid bars) and saccharin (hatched bars) during the same test. $\left.{ }^{* *}\right) P<0.01$ relative to unpaired controls; (\#) $P<0.01$ relative to water intake in SHAM-Paired controls (experiment $3 b$ ). prominent difference between these two studies was the details of the conditioning method.

\section{HISTOLOGY}

Reconstruction of the rostrocaudal extent of excitotoxic amygdala lesions in experiments $3 \mathrm{a}$ (I/O conditioned) and $3 \mathrm{~b}$ (bottle conditioned) are presented on the left and right sides of Figure 8, respectively. Examination of the extent of gliosis revealed that lesioned animals sustained comparable damage to those in experiments 1 and 2 , including the basolateral, lateral, and central amygdaloid nuclei and, to a lesser degree, the ventral aspect of the globus pallidus and striatum.

\section{Experiment 4: Assessment of the Effects of Amygdala Lesions on CTA Learning with the I/O Conditioning Method in Fluid-Deprived Animals}

In the previous experiments, rats with bilateral lesions of amygdala were conditioned either intraorally or with a bottle presentation of the CS taste. However, the fluid deprivation status of the two procedures was not held constant across studies; I/O conditioning does not typically necessitate water deprivation, whereas bottle conditioning does. To control for this procedural difference, the following experiment replicated the I/O procedure of experiment 1 in rats with ibotenic acid lesions of amygdala. Before conditioning, however, these rats were placed on a water deprivation schedule so that that fluid deprivation status was equivalent to that of rats in experiment 2 that were trained with a bottle.

\section{Materials and Methods}

\section{HABITUATION AND CONDITIONING}

Rats with bilateral ibotenic acid lesions of amygdala were assigned to SHAM-paired $(n=6)$, Lesion-paired $(n=4), \quad$ and Lesion-unpaired $(n=4)$ groups and habituated to chambers and $\mathrm{I} / \mathrm{O}$ infusions as described previously. They were then conditioned and tested using the I/O method of experiment 1 . However, these rats were also habituated and conditioned under a water deprivation regimen as in experiment 2 . Thus, their fluid deprivation status at the time of conditioning was comparable to animals in experiments 2 and $3 b$ that were conditioned using a bottle.

$$
\begin{array}{lllllllllllllll}
\hline & E & A & R & N & I & N & G & \begin{array}{c}
\boldsymbol{Q} \\
\mathbf{4 8 8}
\end{array} & M & E & M & O & R & Y
\end{array}
$$




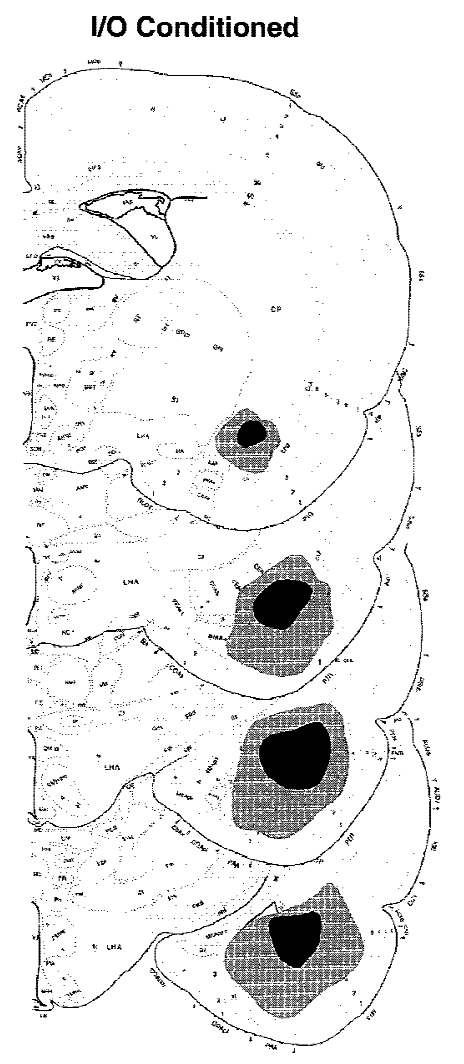

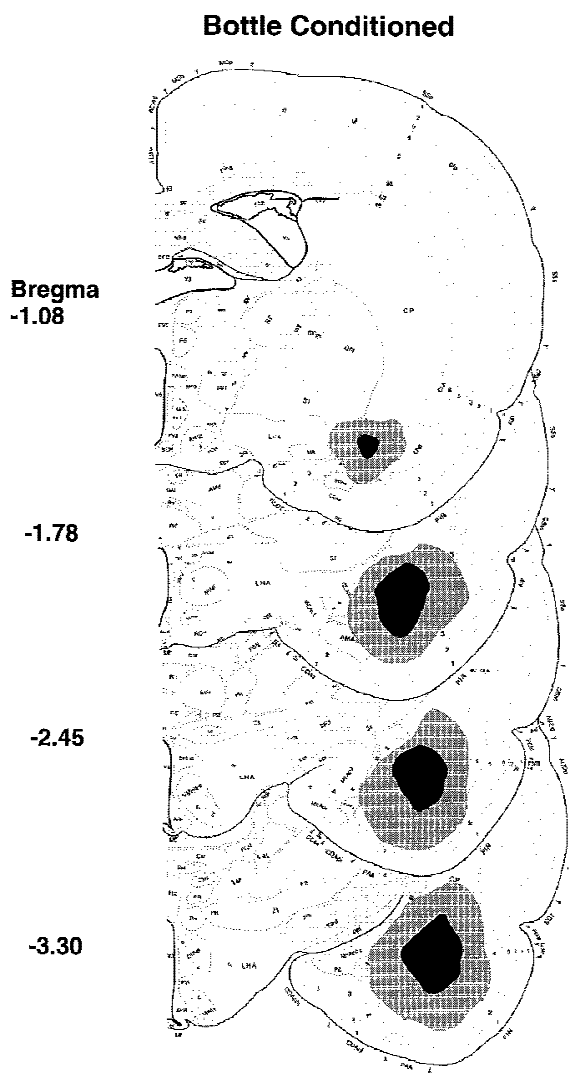

Figure 8: Serial reconstructions of excitotoxic amygdala lesions in the transverse plane (experiment 3). (Left) Lesions in experiment $3 \mathrm{a}$ in which rats were conditioned with the I/O method. (Right) Lesions in experiment $3 \mathrm{~b}$ in which rats were conditioned using a bottle. The lightly and darkly shaded regions correspond to the largest and smallest lesions, respectively.

\section{Results and Discussion}

Mean latency to reject the I/O infusion of CS saccharin in fluid deprived rats with excitotoxic lesions is presented in Figure 9. Clearly, fluid deprivation is not a critical variable influencing whether amygdala lesions disrupt CTA learning. As

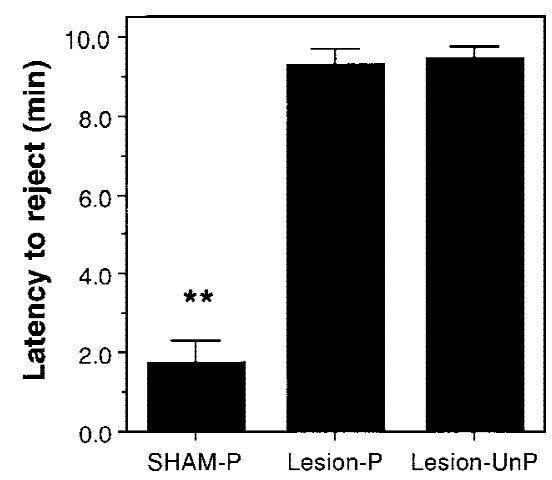

Figure 9: Mean rejection latency ( \pm S.E. $)$ of SHAM and excitotoxically lesioned animals during $\mathrm{I} / \mathrm{O}$ saccharin infusion at the time of testing. Saccharin had either been paired $(\mathrm{P})$ or unpaired $(\mathrm{UnP})$ with $\mathrm{LiCl}$ using $\mathrm{I} / \mathrm{O}$ conditioning under conditions of fluid deprivation. (**) $P<0.01$ relative to lesioned groups (experiment 4 ). in the first experiment, paired rats showed virtually no evidence of aversion conditioning relative to unpaired controls, whereas SHAM animals rejected the CS taste within 2-3 min (Mann-Whitney $U$-test, $P<0.01)$.

\section{GENERAL DISCUSSION}

The role of amygdala in CTA learning has remained controversial, and this controversy has clouded efforts to define the neural pathways critical to this unusual and robust type of learning. The present studies are the first, of which we are aware, to systematically examine the impact of different conditioning methods on the neural mediation of CTA learning. Results suggest a possible reason for the controversy involving amygdala and CTAs, namely that the involvement of amygdala in CTA learning can vary dramatically with the nature of the conditioning method used. Using an I/O CS infusion procedure, the amygdala appeared necessary for CTA expression. Conditioned animals with lesions of the amygdala were indistinguishable from unconditioned controls in their ingestive response to the CS taste. This was the case whether

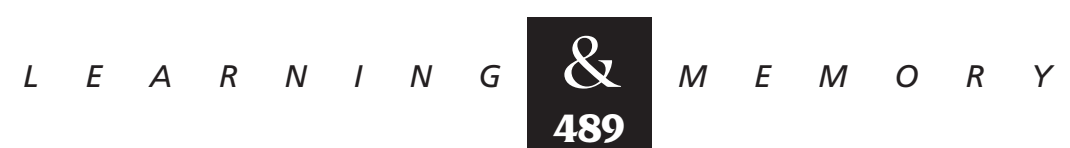


lesions were made electrolytically or using an axon-sparing excitotoxin. In marked contrast, when animals were conditioned in the more conventional way, by receiving CS exposure while drinking from a bottle, effects of amygdala lesions were less dramatic and were only seen with electrolytic lesions.

Most laboratories that study the impact of amygdala lesions on CTA learning utilize bottleconditioning methods that require the animal to voluntarily approach and consume the CS solution before administration of the US drug (e.g., see Nachman and Ashe 1974; Lasiter and Glanzman 1982, 1985; Simbayi et al. 1986; Dunn and Everitt 1988; Bermudez-Rattoni and McGaugh 1991; Gallo et al. 1992; Hatfield et al. 1992; Kesner et al. 1992). The acquisition of CTAs under these circumstances is complex and appears to contain elements of both Pavlovian and instrumental learning (e.g., see Chambers 1990). The taste (CS)-illness (US) contingency, for example, may be characterized procedurally as Pavlovian conditioning, whereas the approach-illness (US) contingency may be characterized as instrumental learning. However, because it has no response requirement, the I/O conditioning method used in the present studies is one in which the Pavlovian components of CTA learning can be isolated from the more conventional method involving an approach component. The neural structures and pathways mediating different types of learning and memory have been shown to be dissociable (McDonald and White 1993). The amygdala, for example, has been shown to be essential for Pavlovian conditioning tasks, particularly aversive tasks (Lavond et al. 1993; McDonald and White 1993; Gallagher and Chiba 1996), whereas the dorsal striatum and hippocampus have been shown to be essential for learning tasks involving either a response or spatial requirement, respectively (e.g., see McDonald and White 1993, 1994, 1995). Consistent with these findings, the results of the present studies strongly imply that the importance of the amygdala to CTA learning depends heavily on whether the method of CS delivery is response contingent or not. When the conditioning procedure does not involve a response component (I/O method), amygdala lesions eliminate CTA acquisition. However, if the conditioning protocol does include a response requirement (bottle method), othermemory systems may be capable of acquisition and performance of the learned response in the amygdala-lesioned animal (McDonald and White 1993, 1994, 1995). Thus, procedural differences, which appear quite subtle, nonetheless apparently result in important differences in the neural circuitry that is recruited to mediate the learning.

Interestingly, the different effects of electrolytic and excitotoxic lesions on CTAs conditioned with the bottle method replicate the widely cited findings of Dunn and Everitt (1988). Using bottletraining methods, they found that electrolytic, but not excitotoxic, lesions of amygdala attenuated CTA learning. They interpreted their results to indicate that the amygdala is not involved in CTA learning and that when electrolytic lesions do affect CTA acquisition it is because of incidental damage to fibers of passage projecting to or originating in structures anterior to the amygdala, such as insular cortex (IC). This is a particularly relevant concern considering that lesions of IC are among the more consistent at eliminating acquisition and/ or retention of CTA learning (Braun et al. 1982; Kiefer et al. 1984; Lasiter and Glanzman 1985; Dunn and Everitt 1988; Bermudez-Rattoni and McGaugh 1991). Consistent with the interpretations of Dunn and Everitt (1988), the present results also suggest a role for fibers of passage in CTA learning but only when the method of CS delivery involves a response requirement.

The strong interpretation of the results of the present studies, particularly the contrast between experiments $3 \mathrm{a}$ and $3 \mathrm{~b}$, is that when taste aversions are conditioned conventionally, using a bottle, amygdala is not involved. On the other hand, when conditioning is accomplished using an I/O CS presentation, amygdala is indispensable. Our resistance to accepting this strong view stems largely from considering evidence from those laboratories that have found effects on CTA learning using traditional training (bottle) methods and the placement of excitotoxic lesions in amygdala (Yamamoto et al. 1995; S. Frey, R. Morris, and M. Petrides, unpubl.). Recent studies, furthermore, have found significant effects on bottle-trained CTAs following the infusion of protein synthesis inhibitors (Lamprecht and Dudai 1996), CREB antisense (Lamprecht and Dudai 196; Lamprecht et al. 1997) or inhibitors of protein kinase C (Yasoshima and Yamamoto 1997) directly into the amygdala, implicating the amygdala in the experience-dependent plastic changes that underlie CTA acquisition. Collectively, this work provides support for a role for amygdala in CTAs when bottle conditioning methods are used. Thus, it is possible that our failure to find effects of excitotoxic lesions

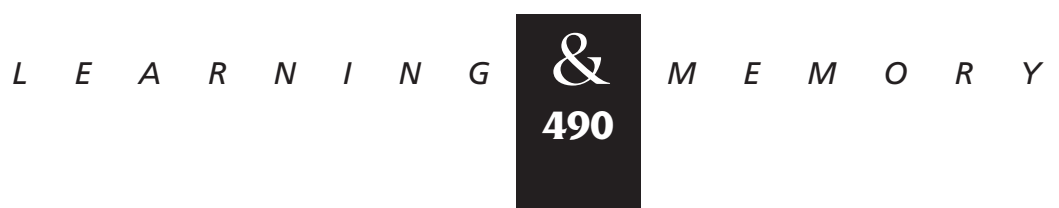


of amygdala on CTAs conditioned with a bottle was attributable ue to insensitivity of our testing methods. The present data certainly provide convincing evidence that the role of amygdala differs markedly depending on the conditioning protocol, but the overall importance of the amygdala in the traditional CTA paradigm remains to be determined.

The clear demonstration that the methods used to condition a taste aversion dramatically affect whether amygdala-lesioned animals are able to demonstrate evidence of CTA learning is important for a number of reasons. Not only does it provide a potential explanation for the long-standing inconsistencies in this literature, but it strongly suggests that the neural circuitry recruited in a CTA learning task, like other learning tasks, varies in its anatomical distribution and complexity depending on key features of the conditioning procedure. These data are consistent with the view that multiple independent memory systems can underlie the acquisition of complex learning tasks (McDonald and White 1993, 1994, 1995). They also provide an interesting parallel to the literature on fear conditioning, another learning task of considerable robustness and adaptive significance, and promise that some of the progress that has been made in understanding the neural basis of fear conditioning can provide a useful model for a similar approach to taste aversion learning. The I/O taste aversion conditioning method has already been shown to have a number of advantages in this regard. We have demonstrated a reliable cellular correlate of a CTA, cFos expression in the iNTS, using this method. This cellular correlate has provided a powerful tool for definition of critical pathways (Schafe et al. 1995; Schafe and Bernstein 1996). Furthermore, the present results suggest that the circuitry critical to this learning paradigm is definable, perhaps because it is simpler and involves less redundancy. Whether the present findings imply that the two different conditioning protocols involve quite different neural circuitry or that there is overlap in circuitry but that the amygdala node is critical to the I/O but not the bottle-trained method remains to be determined. Nonetheless, the present findings necessitate more careful definition of CTA paradigms. It may then be possible to unambiguously address critical questions such as whether amygdaa involvement is primarily in acquisition, retention, or expression of a CTA and whether other parts of the circuit differ as markedly as a function of conditioning protocol, as well as which amygdaloid subnuclei are critical to this involvement.

\section{Acknowledgments}

This research was supported by National Institutes of Health grant NS37040.

The publication costs of this article were defrayed in part by payment of page charges. This article must therefore be hereby marked "advertisement" in accordance with 18 USC section 1734 solely to indicate this fact.

\section{References}

Bermudez-Rattoni, F. and J.L. McGaugh. 1991. Insular cortex and amygdala lesions differentially affect acquisition on inhibitory avoidance and conditioned taste aversion. Brain Res. 549: 165-170.

Bernstein, I.L. 1991. Flavor aversion. In: Smell and taste in health and disease (ed. T.V. Getchell, R.L. Doty, L.M. Bartoshuk, and J.B. Snow), pp. 417-428. Raven Press, New York, NY.

Braun, J.J., P.S. Lasiter, and S.W. Kiefer. 1982. The gustatory neocortex of the rat. Physiol. Psychol. 10: 13-45.

Chambers, K.C. 1990. A neural model of conditioned taste aversions. Annu. Rev. Neurosci. 13: 373-385.

Dunn, L.T. and B.J. Everitt. 1988. Double dissociations of the effects of amygdala and insular cortex lesions on conditioned taste aversion, passive avoidance, and neophobia in rat using the excitotoxin ibotenic acid. Behav. Neurosci. 102: 3-23.

Galaverna, O.G., R.J. Seeley, K.C. Berridge, H.J. Grill, A.N. Epstein, and J. Schulkin. 1993. Lesions of the central nucleus of the amygdala. I: Effects on taste reactivity, taste aversion learning and sodium appetite. Behav. Brain Res. 59: 11-17.

Gallagher, M. and A.A. Chiba. 1996. The amygdala and emotion. Curr. Opin. Neurobiol. 6: 221-227.

Gallo, M., G. Roldan, and J. Bures. 1992. Differential involvement of gustatory insular cortex and amygdala in the acquisition and retrieval of conditioned taste aversion in rats. Behav. Brain Res. 52: 91-97.

Garcia, J., W.G. Hankins, and K.W. Rusiniak. 1994. Behavioral regulation of the milieu interne in man and rat. Science 185: 824-831.

Grigson, P.S., T. Shimura, and R. Norgren. 1997. Brainstem lesions and gustatory function: III. The role of the nucleus of the solitary tract and the parabrachial nucleus in retention of a conditioned taste aversion in rats. Behav. Neurosci. 111: 180-187.

Grill, H.J. and R. Norgren. 1978. Chronically decerebrate rats demonstrate satiation but not bait shyness. Science 201: 267-269.

Hatfield, T., P.W. Graham, and M. Gallagher. 1992. Taste-potentiated odor aversion learning: Role of amygdaloid basolateral complex and central nucleus. Behav. Neurosci. 106: 286-293.

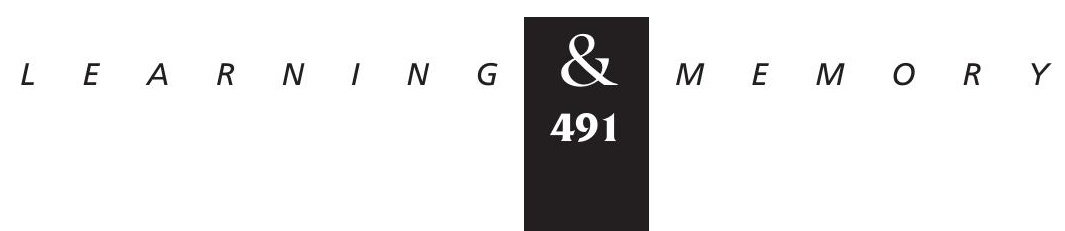




\section{Schafe et al.}

Jarrard, L.E. 1991. Use of ibotenic acid to selectively lesion brain structures. Methods Neurosci. 7: 58-69.

Kesner, R.P., R.F. Berman, and R. Tardif. 1992. Place and taste aversion learning: Role of basal forebrain, parietal cortex, and amygdala. Brain Res.Bull. 29: 345-353.

Kiefer, S.W., L.R. Leach, and J.J. Braun. 1984. Taste agnosia following gustatory neocortex ablation: Dissociation from odor and generality across taste qualities. Behav. Neurosci. 98: $590-608$.

Lamprecht, R. and Y. Dudai. 1996. Transient expression of c-Fos in rat amygdala during training is required for encoding conditioned taste aversion memory. Learn. \& Mem. 3: 31-41.

Lamprecht, R., S. Hazvi, and Y. Dudai. 1997. cAMP response element-binding protein in the amygdala is required for longbut not short- term conditioned taste aversion memory. J. Neurosci. 17: 8443-8450.

Lasiter, P.S. and D.L. Glanzman. 1982. Cortical substrates of taste aversion learning: Dorsal prepiriform (insular) lesions disrupt taste aversion learning. Brain Res. Bull. 29: 345-353.

1985. Cortical substrates of taste aversion learning: Involvement of dorsolateral amygdaloid nuclei and temporal neocortex in taste aversion learning. Behav. Neurosci. 99: $257-276$.

Lavond, D.G., J.J. Kim, and R.F. Thompson. 1993. Mammalian brain substrates of aversive classical conditioning. Annu. Rev. Psychol. 44: 317-342.

McDonald, R.J. and N.M. White. 1993. A triple dissociation of memory systems: Hippocampus, amygdala, and dorsal striatum. Behav. Neurosci. 107: 3-22.

1994. Parallel information processing in the water maze: Evidence for independent memory systems involving dorsal striatum and hippocampus. Behav. Neural Biol. 61: $260-270$.

1995. Hippocampal and nonhippocampal contributions to place learning in rats. Behav. Neurosci. 109: 579-593.

Nachman, M. and J.H. Ashe. 1974. Effects of basolateral amygdala lesions on neophobia, learned taste aversions, and sodium appetite in rats. J. Comp. Physiol. Psychol. 87: 622-643.

Paxinos, G. and C. Watson. 1986. The rat brain in stereotaxic coordinates, 2nd ed, Academic Press, Orlando. FL.

Reilly, S., P.S. Grigson, and R. Norgren. 1993. Parabrachial nucleus lesions and conditioned taste aversion: Evidence supporting an associative deficeit. Behav. Neurosci. 107: 1005-1017.

Riley, A.L. and C.M. Clarke. 1977. Conditioned taste aversions: A bibliography. In Learning mechanisms in food selection (ed. L.M. Barker, M.R. Best, M. Domjan). Baylor University Press, Waco, TX.

Schafe, G.E. and I.L. Bernstein. 1996. Forebrain contribution to the induction of a brainstem correlate of conditioned taste aversion: I. The amygdala. Brain Res. 741: 109-116.

Schafe, G.E., R.J. Seeley, and I.L. Bernstein. 1995. Forebrain contribution to the induction of a cellular correlate of conditioned taste aversion in the nucleus of the solitary tract. J. Neurosci. 15: 6789-6796.

Simbayi, L.C., R.A. Boakes, and M.J. Burton. 1986. Effects of basolateral amygdala lesions on taste aversion produced by lactose and lithium chloride in the rat. Behav. Neurosci. 100: 455-465.

Swank, M.W. and I.L. Bernstein. 1994. cFos induction in response to a conditioned stimulus after single trial taste aversion learning. Brain Res. 636: 202-208.

Swank, M.W., G.E. Schafe, and I.L. Bernstein. 1995. c-Fos induction in response to taste stimuli previously paired with amphetamine or $\mathrm{LiCl}$ during taste aversion learning. Brain Res. 673: 251-261.

Swanson, L.W. 1994. Brain maps: Computer graphics files (version 1.0). Elsevier, Amsterdam, The Netherlands.

Yamamoto, T. 1993. Neural mechanisms of taste aversion learning. Neurosci. Res. 16: 181-185.

Yamamoto, T. and Y. Fujimoto. 1991. Brain mechanisms of taste aversion learning in the rat. Brain Res. Bull. 27: 403-406.

Yamamoto, T., Y. Fujimoto, T. Shimura, and N. Sakai. 1995. Conditioned taste aversion in rats with excitotoxic brain lesions. Neurosci. Res. 1: 31-49.

Yasoshima, Y. and T. Yamamoto. 1997. Rat gustatory memory requires protein kinase $\mathrm{C}$ activity in the amygdala and cortical gustatory area. Neuroreport 8: 1363-1367.

Received August 10, 1998; accepted in revised form October 28, 1998.

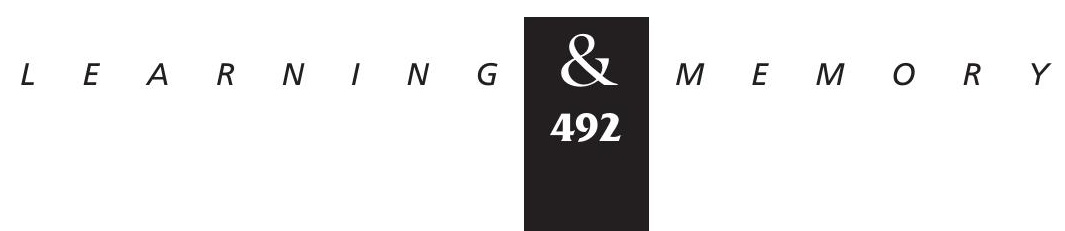




\section{Errata}

Learning \& Memory 5:481-492 (1998)

Conditioning Method Dramatically Alters the Role of Amygdala in Taste Aversion Learning Glenn E. Schafe, Todd E. Thiele, and Ilene L. Bernstein

Because of problems in production, several errors were retained in this article. In the legend to Figure 6 , the single asterisk should be replaced by a double asterisk. On page 491, the following sentence should have been deleted: "They also provide an interesting parallel to the literature on fear conditioning, another learning task of considerable robustness and adaptive significance, and promise that some of the progress that has been made in understanding the neural basis of fear conditioning can provide a useful model for a similar approach to taste aversion learning."

Learning \& Memory 5:467-480 (1998)

On the Respective Roles of Nitric Oxide and Carbon Monoxide in Long-Term Potentiation in the Hippocampus

Min Zhuo, Jarmo T. Laitinen, Xiao-Ching Li, and Robert D. Hawkins

Because of problems in production, many errors were retained in this article. It is reprinted in its entirety on the following pages.

LEARNING \& MEMORY 6:62 @ 1999 by Cold Spring Harbor Laboratory Press ISSN1072-0502/99 \$5.00

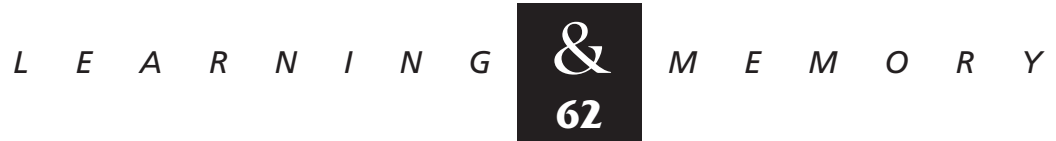




\title{
On the Respective Roles of Nitric Oxide and Carbon Monoxide in Long-Term Potentiation in the Hippocampus
}

\author{
and Robert D. Hawkins ${ }^{1,3,5}$ \\ ${ }^{1}$ Center for Neurobiology and Behavior \\ College of Physicians and Surgeons of Columbia University \\ ${ }^{2}$ Howard Hughes Medical Institute \\ and ${ }^{3}$ New York State Psychiatric Institute \\ New York, New York 10032 USA \\ ${ }^{4}$ Department of Physiology \\ University of Kuopio \\ FIN-70211 \\ Kuopio, Finland
}

Min Zhuo, ${ }^{1,2,6}$ Jarmo T. Laitinen, ${ }^{4}$ Xiao-Ching Li, ${ }^{1,2}$

\section{Abstract}

Perfusion of hippocampal slices with an inhibitor nitric oxide (NO) synthase blocked induction of long-term potentiation (LTP) produced by a one-train tetanus and significantly reduced LTP by a two-train tetanus, but only slightly reduced LTP by a four-train tetanus. Inhibitors of heme oxygenase, the synthetic enzyme for carbon monoxide (CO), significantly reduced LTP by either a two-train or four-train tetanus. These results suggest that $\mathrm{NO}$ and $\mathrm{CO}$ are both involved in LTP but may play somewhat different roles. One possibility is that NO serves a phasic, signaling role, whereas $\mathrm{CO}$ provides tonic, background stimulation. Another possibility is that NO and $\mathrm{CO}$ are phasically activated under somewhat different circumstances, perhaps involving different receptors and second messengers. Because NO is known to be activated by stimulation of NMDA receptors during tetanus, we investigated the possibility that $\mathrm{CO}$ might be activated by stimulation of metabotropic glutamate receptors (mGluRs). Consistent with this

\footnotetext{
${ }^{5}$ Corresponding author.

${ }^{6}$ Present address: Department of Anesthesiology, Washington University, St. Louis, Missouri 63110 USA.
}

idea, long-lasting potentiation by the mGluR agonist tACPD was blocked by inhibitors of heme oxygenase but not NO synthase. Potentiation by tACPD was also blocked by inhibitors of soluble guanylyl cyclase (a target of both NO and $\mathrm{CO}$ ) or cGMP-dependent protein kinase, and guanylyl cyclase was activated by tACPD in hippocampal slices. However, biochemical assays indicate that whereas heme oxygenase is constitutively active in hippocampus, it does not appear to be stimulated by either tetanus or tACPD. These results are most consistent with the possibility that constitutive (tonic) rather than stimulated (phasic) heme oxygenase activity is necessary for potentiation by tetanus or tACPD, and suggest that mGluR activation stimulates guanylyl cyclase phasically through some other pathway.

\section{Introduction}

Long-term potentiation (LTP) is a sustained increase in synaptic efficacy that is thought to be one of the candidate mechanisms for memory storage in the hippocampus (for reviews, see Bliss and Collingridge 1993; Hawkins et al. 1993). In the CA1 region of hippocampus, the induction of LTP generally requires $\mathrm{Ca}^{2+}$ influx through postsynaptic $N$ methyl-D-aspartate (NMDA) glutamate receptor

LEARNING \& MEMORY 5:63-76 @ 1999 by Cold Spring Harbor Laboratory Press ISSN1072-0502/99 \$5.00

$$
\begin{array}{lllllllllllllll}
L & E & A & R & N & I & N & G & \underset{63}{\boldsymbol{Z}} & M & E & M & O & R & Y
\end{array}
$$


channels, but the maintenance of LTP is thought to involve, in part, a presynaptic enhancement of transmitter release. These results suggest that the postsynaptic cells must send one or more retrograde messengers to the presynaptic terminals. There is now evidence that several molecules may act as such retrograde messengers during LTP in hippocampus, including the soluble gases nitric oxide (NO) (Böhme et al. 1991; O'Dell et al. 1991; Schuman and Madison 1991; Haley et al. 1992; Zhou et al. 1993; Arancio et al. 1996) and carbon monoxide (CO) (Stevens and Wang 1993; Zhuo et al. 1993), as well as arachidonic acid (Williams et al. 1989), platelet-activating factor (Del Cerro et al. 1990; Clark et al. 1992; Wieraszko et al. 1993), and several neurotrophins (Kang and Schuman 1995; Thoenen 1995; Korte et al. 1996). However, a number of questions remain concerning the possible roles of NO and CO. First, although several studies have found that inhibitors, targeted mutation, or adenovirus-mediated inhibition of NO synthase block the induction of LTP in CA1 and dentate gyrus (Böhme et al. 1991; O'Dell et al. 1991; Schuman and Madison 1991; Haley et al. 1992; Mizutani et al. 1993; Boulton et al. 1995; Doyle et al. 1996; Son et al. 1996; Kantor et al. 1996; Wu et al. 1997), other studies have found that inhibitors of NO synthase either do not block LTP (Kato and Zorumski 1993; Bannerman et al. 1994; Cummings et al. 1994) or block LTP only under some experimental circumstances and not others (Gribkoff and Lum-Ragan 1992; Chetkovich et al. 1993; Haley et al. 1993, 1996; Williams et al. 1993; O’Dell et al. 1994; Malen and Chapman 1997). Second, inhibitors of heme oxygenase (the synthetic enzyme for CO) can also block the induction of LTP in the CA1 region of hippocampus and dentate gyrus (Stevens and Wang 1993; Zhuo et al. 1993; Ikegaya et al. 1994), but there are concerns about the specificity of those inhibitors (Ignarro et al. 1984; Linden et al. 1993; Luo and Vincent 1994; Meffert et al. 1994; Okada 1996). Third, if, as the inhibitor studies suggest, NO and CO are both involved in LTP, it is not clear what their respective roles might be. One possibility is that NO and $\mathrm{CO}$ are activated under somewhat different circumstances. This possibility would be consistent with the finding that inhibitors of NO synthase do not block LTP under all circumstances, suggesting that the residual potentiation might be mediated by another messenger. Alternatively, CO may provide a tonic level of stimulation, whereas NO provides phasic stimulation during the induction of LTP. This possibility would be consistent with the fact that NO has a very short half-life, whereas $\mathrm{CO}$ is more stable.

In the present study, we have addressed these questions to try to clarify the possible roles of NO and CO in LTP.

\section{Materials and Methods}

\section{ELECTROPHYSIOLOGY}

Male 4- to 6-week-old Sprague-Dawley rats were housed and sacrificed in accordance with the guidelines of the Health Sciences Division of Columbia University. Transverse slices of hippocampus $(400 \mu \mathrm{M})$ were rapidly prepared and maintained between 28 and $30^{\circ} \mathrm{C}$ in an interface chamber (Fine Science Tools, Foster City, CA), in which they were subfused with artificial cerebrospinal fluid (ACSF) consisting of: $124 \mathrm{~mm} \mathrm{NaCl}, 4.0 \mathrm{~mm}$ $\mathrm{KCl}, 2.0 \mathrm{~mm} \mathrm{CaCl}_{2}, 2.0 \mathrm{~mm} \mathrm{MgSO}_{4}, 1.0 \mathrm{~mm}$ $\mathrm{Na}_{2} \mathrm{HPO}_{4}, 24.1 \mathrm{~mm} \mathrm{NaHCO}, 10 \mathrm{~mm}$ glucose, bubbled with $95 \% \mathrm{O}_{2}$ and $5 \% \mathrm{CO}_{2}$. Slices were allowed to recover for at least $1.5 \mathrm{hr}$ before experiments were performed. A bipolar tungsten stimulating electrode was placed in the stratum radiatum of the CA1 region or in the stratum pyramidale of the CA3 region. In some experiments, a second independent stimulating electrode was placed in the stratum radiatum of the CA1 region on the other side of the recording microelectrode. Before the start of the experiments, paired-pulse facilitation (50 ms interval) was tested to verify the independence of the two pathways. Extracellular field potentials were recorded with a glass microelectrode (3-12 $\mathrm{m} \Omega$ filled with ACSF) placed in the stratum radiatum. The stimulation intensity was adjusted to give field EPSP amplitudes of 1.0-1.5 mV (which is $\sim 25 \%$ of maximum) so that the weak tetanus by itself would not produce LTP. The two pathways were stimulated alternately at $0.02 \mathrm{~Hz}$ and the initial slope of the EPSP was measured. If the recording was stable for at least $30 \mathrm{~min}$, potentiation was produced in one pathway by use of one of four tetanic stimulation protocols; (1) one train of $100 \mathrm{~Hz}, 1 \mathrm{sec}$ stimulation; (2) two trains of 100 $\mathrm{Hz}, 1 \mathrm{sec}$ stimulation separated by $20 \mathrm{sec}$; (3) four trains of $100 \mathrm{~Hz}, 1 \mathrm{sec}$ stimulation separated by 5 min; or (4) one train of $50 \mathrm{~Hz}, 0.5 \mathrm{sec}$ stimulation (weak tetanus) during brief ( $2 \mathrm{~min}$ ) perfusion with CO, tACPD, or 8-Br-cGMP. Current intensity during the tetanic stimulation was always the same as test intensity. Potentiation was measured in each experiment as the average EPSP slope 50-60 min

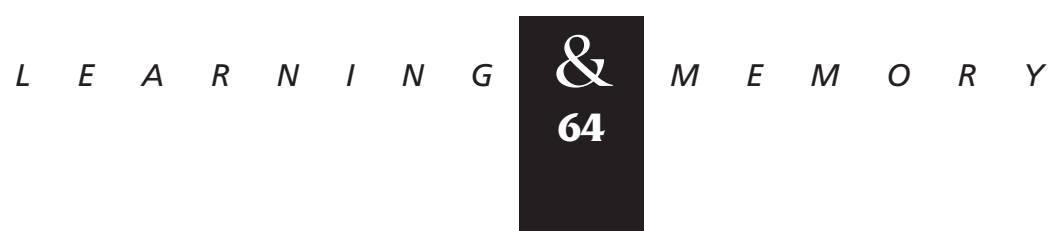


post-tetanus as a percentage of the average baseline for $30 \mathrm{~min}$ pre-tetanus.

\section{DRUG PREPARATION}

$N^{\omega}$-nitro-arginine, AP3, and 8-Br-cGMP (Sigma, St. Louis, MO), tACPD (RBI, Natick, MA), and Rp8-Br-cGMPS (Biolog, La Jolla, CA) were dissolved in ACSF immediately before each experiment. Zinc protoporphyrin IX, tin protoporphyrin IX, copper protoporphyrin, and zinc deuteroporphyrin IX-3,4bis-glycol (Porphyrin Products, Logan, UT) and LY83583 (Biomol, Plymouth Meeting, PA) were dissolved in dimethyl sulfoxide (DMSO) immediately before each experiment and diluted to the desired concentration in ACSF by sonication (final concentration of DMSO was $\leqslant 0.05 \%$ ). (+)-MCPG (Tocris Neuramin, Bristol, UK) was dissolved with the 1.1 equivalent of sodium hydroxide solution to give $100 \mathrm{~mm}$ stock solution and then diluted to the desired concentration in ACSF. Hemoglobin was prepared from methemoglobin as described by Martin et al. (1985). CO solution was prepared by bubbling the gas in distilled water until saturation and immediately diluted to the desired concentration in ACSF. Slices were perfused with the various inhibitors throughout the experiment, starting at least $30 \mathrm{~min}$ before the beginning of the baseline period. CO, tACPD, or 8-Br-cGMP were injected directly into the recording chamber for $2 \mathrm{~min}$ before the weak tetanic stimulation and then washed out over a period of 5-10 $\mathrm{min}$.

\section{CGMP ASSAY}

Hippocampal slices were prepared as in the LTP experiments and rested at $30^{\circ} \mathrm{C}$ for $2 \mathrm{hr}$ with continuous ACSF perfusion. The slices were pretreated with $200 \mu \mathrm{M}$ IBMX for 20 min followed by $100 \mu \mathrm{M}$ tACPD, $10 \mu \mathrm{M}$ NMDA, and $300 \mu \mathrm{M}$ SNP or ACSF for $\sim 2 \mathrm{~min}$. The slices were then removed from the perfusion chamber and frozen within 10 sec. After the CA3 region was removed, the slices were homogenized in 6\% trichloroacetic acid. Protein was precipitated by spinning at $2000 \mathrm{~g}$ for 20 min, and then the supernatant was extracted four times with water-saturated ether and dried under vacuum. The amount of cGMP in each sample was measured by radioimmunoassay (NEN) following the manufacturer's instructions. The precipitated protein was dissolved in $100 \mathrm{~mm} \mathrm{NaOH}$ and $0.3 \%$ SDS and quantified with the BCA protein assay kit
(Pierce). The cGMP level in each slice was normalized to protein. There were three slices per condition in each experiment, and the average cGMP level for the experimental slices was expressed as a percentage of the average level for the control slices in that experiment. All of the slices in one experiment came from the same animal.

\section{HEME OXYGENASE ACTIVITY ASSAY}

Following in vitro treatment, hippocampal slices were frozen rapidly in dry ice. Tissue samples from the CA1 region of the hippocampus were collected after removing the CA3 region and dentate gyrus. To obtain enough material to assay, three slices were pooled together. Tissue samples were shipped to Finland on dry ice for heme oxygenase activity measurements, which were performed blind to the experimental treatment. Enzyme activity was determined by use of a novel sensitive microassay that relies on the conversion of $\left[{ }^{14} \mathrm{C}\right]$ heme to $\left[{ }^{14} \mathrm{C}\right]$ bilirubin by the concerted activity of heme oxygenase, NADPH-cytochrome $\mathrm{P}-450$ reductase and biliverdin reductase, as described previously (Laitinen and Juvonen 1995). Briefly, slices (3-4 per assay) were sonicated at $0^{\circ} \mathrm{C}$ in $50 \mu$ of 0.1 M K-phosphate buffer ( $\mathrm{pH} 7.5$ ) containing $50 \mu \mathrm{m}$ phenylmethyl sulfonyl fluoride. The homogenate was centrifugated at $14,000 \mathrm{~g}$ for 1 min in an Eppendorf minifuge. Duplicate aliquots of the supernatant ( $5 \mu \mathrm{l} / 7-26 \mu \mathrm{g}$ protein) were incubated in $0.1 \mathrm{M}$ K-phosphate buffer at $\mathrm{pH} 7.5$ (total volume $10 \mu \mathrm{l}$ ) containing $\left[{ }^{14} \mathrm{C}\right]$ heme (sp. act. $52.5 \mathrm{Ci} / \mathrm{mole})$ and NADPH $(2 \mathrm{~mm})$. The final substrate concentration was $21.4 \mu \mathrm{M}$ in all but one experiment in which $4.4 \mu \mathrm{M}$ substrate concentration was used to test for possible liberation of endogenous competing substrates during strong tetanic stimulation. Reagent blanks contained buffer instead of NADPH. Following $15 \mathrm{~min}$ incubation at $37^{\circ} \mathrm{C}$, the tubes were cooled to $0^{\circ} \mathrm{C}$ and $190 \mu \mathrm{l}$ of ice-cold K-phosphate buffer was added. $\left[{ }^{14} \mathrm{C}\right]$ bilirubin was extracted into toluene and counted in a Wallac LKB 1214 Rackbeta with 95.5\% counting efficiency. Heme oxygenase activity (reagent blanks subtracted) is expressed as picomoles of $\left[{ }^{14} \mathrm{C}\right]$ bilirubin formed/mg protein per hour and was corrected for the extraction efficiency $(15.4 \pm 0.4 \%, n=14)$. The following criteria have been applied to validate this method for the measurement of heme oxygenase activity in the rat brain (Laitinen and Juvonen 1995). First, incubation of rat brain homogenate with $\left[{ }^{14} \mathrm{C}\right]$ heme

$$
\begin{array}{llllllllllllllll}
L & E & A & R & N & I & N & G & \underset{65}{\mathbf{Z}} & M & E & M & O & R & Y
\end{array}
$$


yielded a single reaction product, indistinguishable from bilirubin by thin layer chromatography. Second, the reaction was totally dependent on the presence of NADPH, was not catalyzed by boiled tissue, and was inhibited by ZnPP at doses known to inhibit purified rat heme oxygenase $\left(\mathrm{IC}_{50}=0.3\right.$ $\mu \mathrm{M})$. Third, the reaction required the presence of NADPH-cytochrome P-450, as evidenced by the ability of an antibody against the reductase to inhibit the reaction in a dose-dependent fashion.

\section{DATA ANALYSIS AND STATISTICS}

All data are presented as the mean \pm s.E.M. The field EPSPs are presented as percentage of baseline. Statistical comparisons were made by by use of Student's $t$-tests for comparison of groups or paired data. In all cases, $P<0.05$ was considered significant.

\section{Results}

EFFECTS OF AN INHIBITOR OF NO SYNTHASE ON LTP INDUCED BY DIFFERENT NUMBERS OF TETANI

NO synthase inhibitors have blocked the induction of LTP in some studies, but have failed to block LTP in other studies. These discrepancies might be partially explained by different experimental conditions, including the strength of the tetanic stimulation (Gribkoff and Lum-Ragan, 1992; Chetkovich et al. 1993; Haley et al. 1993; O'Dell et al. 1994; Malen and Chapman 1997). To investigate this possibility further, we systematically examined the effects of inhibiting NO synthase on LTP induced by different numbers of tetanic stimuli. We found that pretreatment with the NO synthase inhibitor $N^{\omega}$-nitro-arginine $(100 \mu \mathrm{M})$ for at least 30 min completely abolished LTP produced by a onetrain tetanus (Fig. 1A). $N^{\omega}$-nitro-arginine also significantly reduced LTP produced by a two-train tetanus $(130.8 \pm 23.9 \%, n=10$ compared with $199.4 \pm 18.6 \%, n=10$ in normal saline, $t=2.27$, $P<0.05)$ and completely blocked it in 6 of 10 experiments. But this inhibitor produced only a slight reduction of LTP that was not significant when a four-train tetanus was used (Fig. 1B). These results suggest that NO makes a substantial contribution to the induction of LTP produced with low or moderate tetanic stimulation, but that other messengers may contribute more to LTP induced by stronger tetanic stimulation.
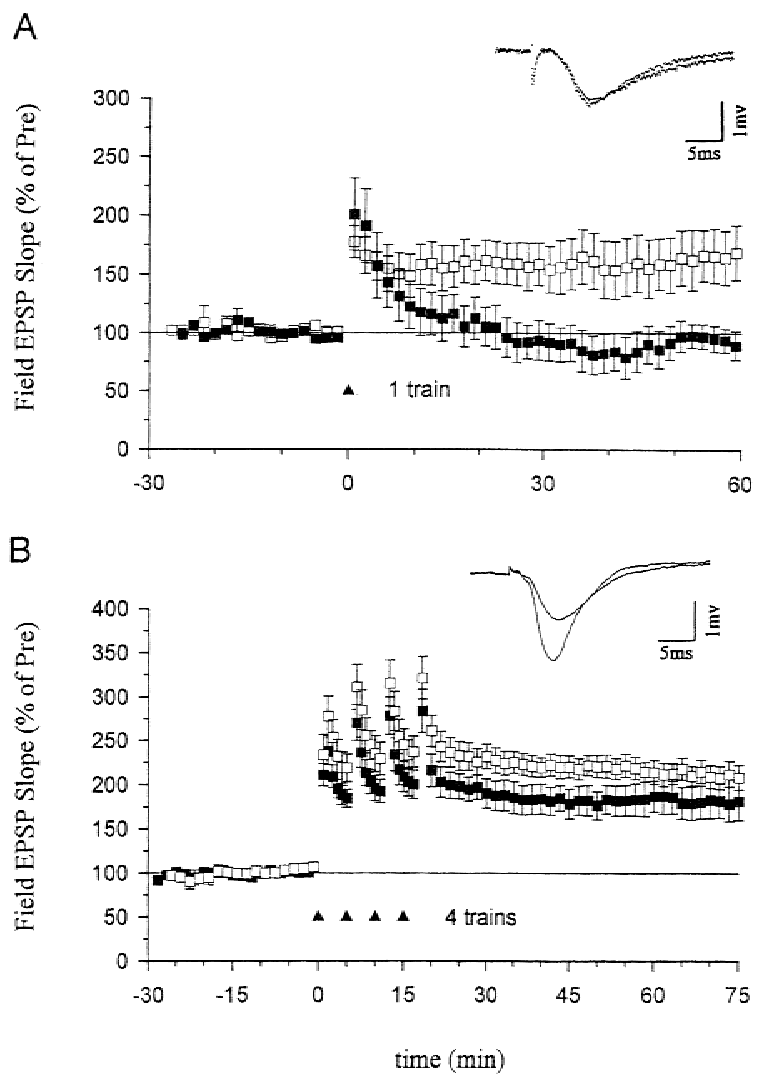

Figure 1: Effects of an inhibitor of nitric oxide synthase on LTP induced by a one- or four-train tetanus. (A) $N^{\omega_{-}}$ nitro-arginine $(100 \mu \mathrm{M})$, a nitric oxide synthase inhibitor, blocked LTP induced by a one-train tetanus $(100 \mathrm{~Hz}$, $1 \mathrm{sec}$ ) (normal ACSF, average EPSP slope 50-60 min post-tetanus $=162.7 \pm 22.7 \%$ of the average baseline for $30 \mathrm{~min}$ pre-tetanus, $n=8 ; N^{\omega}$-nitro-arginine, $94.1 \pm 11.4 \%, n=6, t=2.42, P<0.05$ compared with ACSF-treated slices). (Inset) Representative recordings of the field EPSP before and 60 min after a one-train tetanus in a slice pretreated with $N^{\omega}$-nitro-arginine. (B) $N^{\omega}$-nitro-arginine $(100 \mu \mathrm{M})$ only slightly reduced LTP induced by a four-train tetanus (four $1-\mathrm{sec} 100-\mathrm{Hz}$ trains delivered at an interval of $5 \mathrm{~min} ; 181.0 \pm 20.3 \%, n=7$ ) as compared with ACSF-treated slices $(210.5 \pm 12.9 \%$, $n=9$, not significantly different). (Inset) Representative recordings of the field EPSP before and 60 min after a four-train tetanus in a slice pretreated with $N^{\omega}$-nitroarginine. The average prevalues were $0.45 \mathrm{mV} / \mathrm{msec}$ (ACSF), 0.40 ( $N^{\omega}$-nitro-arginine) $(A)$ and 0.41 (ACSF), 0.36 ( $N^{\omega}$-nitro-arginine) (B). ( $\square$ ) ACSF; ( $\square$ ) Nitro-arginine.

\section{TESTS OF INHIBITORS OF HEME OXYGENASE}

Another candidate retrograde messenger is CO. Inhibitors of heme oxygenase (the synthetic enzyme for CO) block the induction of LTP, and CO paired with weak tetanic or low-frequency

$$
\begin{array}{llllllllllllllll} 
& E & A & R & N & I & N & G & \begin{array}{l}
\boldsymbol{Q} \\
66
\end{array} & M & E & M & O & R & Y
\end{array}
$$


stimulation produces long-lasting potentiation (Stevens and Wang 1993; Zhuo et al. 1993). However, heme oxygenase inhibitors may also act by inhibiting other enzymes, including NO synthase (Meffert et al. 1994; Okada 1996). To examine whether inhibition of NO synthase, rather than heme oxygenase, might account for the block of LTP, we compared the effectiveness of inhibitors of heme oxygenase and NO synthase on LTP induced by different numbers of tetanic stimuli.

LTP induced by two trains of tetanus was significantly attenuated by the heme oxygenase in-

\section{A}

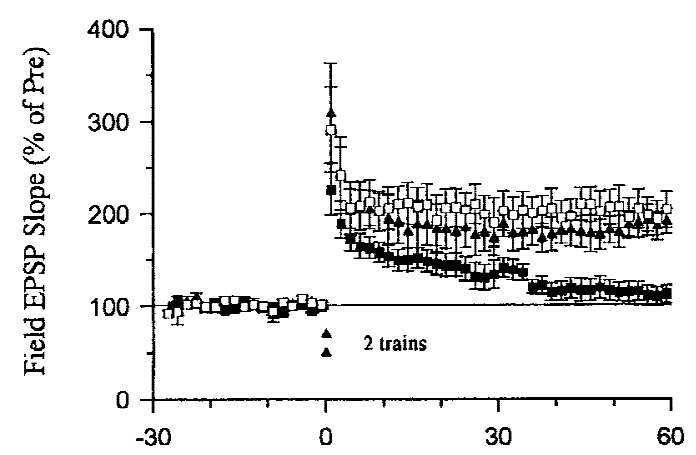

C

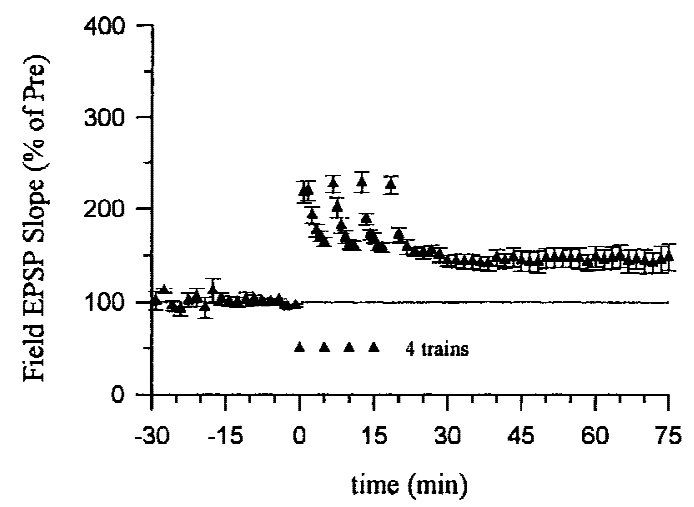

hibitors Tin protoporphyrin IX (SnPP, $10 \mu \mathrm{M})$ or zinc-deuteroporphyrin IX-2,4-bis-glycol (ZnBG, 10 $\mu \mathrm{M}$ ) (Fig. 2A). In contrast, an inactive analog, copper protoporphyrin (CuPP, $10 \mu \mathrm{M})$, did not significantly affect LTP. LTP produced by a four-train tetanus was also significantly reduced by ZnBG (10 $\mu \mathrm{M}$, Fig. 2B) or zinc protoporphyrin (ZnPP, $10 \mu \mathrm{M}$, $152.1 \pm 17.5 \%, n=6 ; t=2.74, P<0.05$ compared with normal LTP induced by a four-train tetanus). Heme oxygenase inhibitors did not completely block the potentiation induced by a four-train tetanus, however. To test whether the remaining po-

B
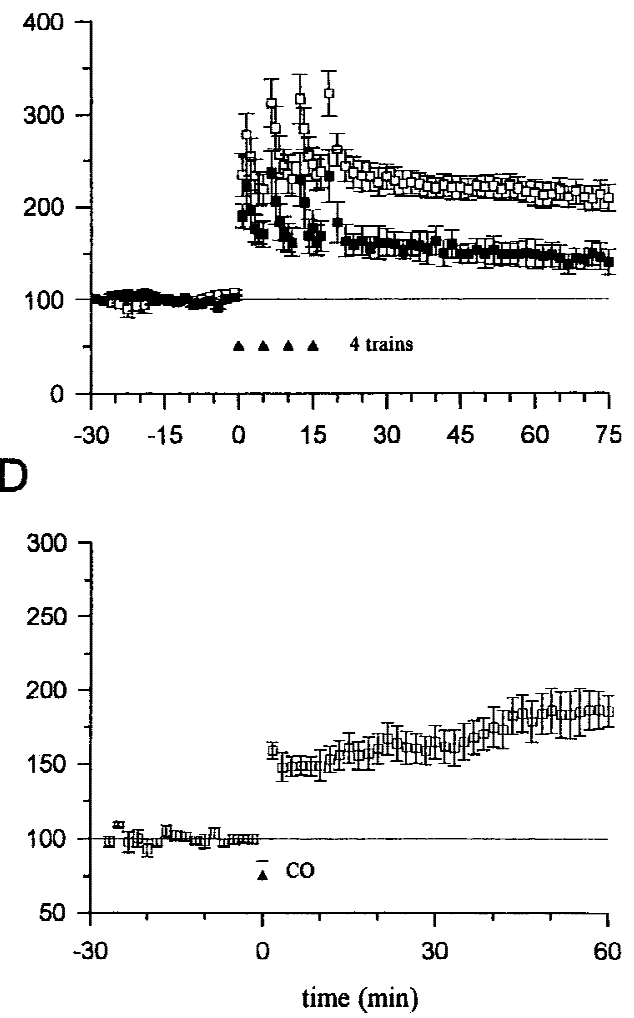

Figure 2: Effects of inhibitors of heme oxygenase on LTP induced by a two- or four-train tetanus. ( $A$ ) Average potentiation of the field EPSP by a two-train tetanus $(100 \mathrm{~Hz}$ for $1 \mathrm{sec}$ each, separated by $20 \mathrm{sec})$ in normal ACSF and in ACSF containing $10 \mu \mathrm{M}$ tin protoporphyrin IX (SnPP), zinc-deuteroporphyrin IX-2,4-bis-glycol (ZnBG), or copper protoporphyrin (CuPP). Perfusion with SnPP, ZnBG, or CuPP started at least 30 min before the tetanus (normal LTP, 199.4 $\pm 18.6 \%$, $n=10 ; 10 \mu \mathrm{M}$ SnPP, $129.1 \pm 3.8 \%, n=7, t=2.79, P<0.05$ compared with normal LTP; $10 \mu \mathrm{M}$ ZnBG, $126.8 \pm 8.3 \%$, $n=6, t=2.88, P<0.05$ compared with normal LTP; $10 \mu \mathrm{M}$ CuPP, $188.1 \pm 14.3 \%, n=5)$. Results with SnPP and ZnBG were similar and have been pooled. $(\square)$ ACSF; $(\mathbf{\Lambda})$ CuPP; $(\mathbf{\square})$ SnPP/ZnBG. (B) Average potentiation by a four-train tetanus in $\operatorname{ACSF}(210.5 \pm 12.9 \%, n=9)$ and in ACSF containing ZnBG $(10 \mu \mathrm{M}, 145.5 \pm 14.2 \%, n=6, t=3.18, P<0.01$ compared with saline-treated slices). ( $\square$ ) ACSF; ( $\square$ ) ZnBG. (C) Average potentiation by a four-train tetanus in ACSF containing both ZnBG $(10 \mu \mathrm{M})$ and $N^{\omega}$-nitro-arginine $(100 \mu \mathrm{M})(143.1 \pm 12.6 \%, n=5)$. (D) ZnPP-IX $(10 \mu \mathrm{M})$ or ZnBG $(10 \mu \mathrm{M})$ did not block long-term enhancement produced by CO (100 nm) paired with weak tetanic stimulation (ZnPP-IX, 160.8 $\pm 19.9 \%, n=5$; $\mathrm{ZnBG}, 236.0 \pm 22.7 \%, n=5)$. Results with the two inhibitors were similar and have been pooled. The average prevalues were $0.37 \mathrm{mV} / \mathrm{msec}$ (normal LTP), 0.38 (SnPP), 0.40 (ZnBG) and 0.39 (CuPP) (A); 0.45 (ACSF), 0.46 (ZnBG) (B); 0.34 (C); and $0.29(D)$.

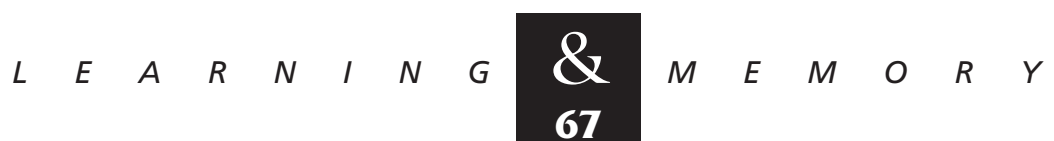


tentiation is mediated by NO, we pretreated slices with both $N^{\omega}$-nitro-arginine (100 $\left.\mu \mathrm{M}\right)$ and ZnBG (10 $\mu \mathrm{M})$. The added NO synthase inhibitor did not produce further reduction as compared with $\mathrm{ZnBG}$ alone (Fig. 2C). Thus, LTP induced by four trains of stimulation was only slightly reduced by an inhibitor of NO synthase, but was significantly reduced by inhibitors of heme oxygenase at a 10-fold lower dosage. Moreover, the combination of an NO synthase inhibitor and a heme oxygenase inhibitor had effects similar to the heme oxygenase inhibitor alone but not the NO synthase inhibitor alone. These results suggest that heme oxygenase inhibitors did not act by inhibiting NO synthase in these studies.

Another potential concern is that inhibitors of heme oxygenase might also act by inhibiting soluble guanylyl cyclase (Ignarro et al. 1984; Luo and Vincent 1994), which could block the induction of LTP (Zhuo et al. 1994; Boulton et al. 1995; Son et al. 1998). To test this possibility, we examined enhancement by $\mathrm{CO}$, which stimulates soluble guanylyl cyclase (J.T. Laitinen, K.S.M. Laitinen, L. Tuomiste, and M.M. Airaksinenm, unpubl; Maines 1993; Verma et al. 1993). If the heme oxygenase inhibitors act by directly inhibiting guanylyl cyclase, they should block the potentiation produced by CO. In the presence of $10 \mu \mathrm{M} \mathrm{ZnPP}$ or ZnBG, CO (100 nm) paired with weak stimulation still produced long-lasting potentiation (Fig. 2D). These observations suggest that ZnPP or ZnBG at a dose of $10 \mu \mathrm{M}$ did not inhibit soluble guanylyl cyclase in these experiments, but rather acted by inhibiting heme oxygenase. These findings are consistent with recent studies showing that inhibitors of heme oxygenase at this dose have little effect on NO synthase or soluble guanylyl cyclase in endothelial or intestinal tissue (Zakhary et al. 1996, 1997).

\section{EFFECTS OF HEME OXYGENASE INHIBITORS ON TACPD-INDUCED ENHANCEMENT}

The results of the inhibitor experiments suggest that NO and CO may both be involved in LTP, which raises the question of what their respective roles might be. One possibility suggested by these results is that NO synthase and heme oxygenase are activated by different stimulation patterns that might engage different receptors and second messengers. In the hippocampus, NO synthase is activated by stimulation of NMDA glutamate receptors during tetanic stimulation (East and Garthwaite
1991; Chetkovich et al. 1993). Heme oxygenase is present in hippocampal pyramidal cells (Maines 1993; Verma et al. 1993), but it is not known whether heme oxygenase can be acutely activated during tetanic stimulation. Heme oxygenase appears to be activated by stimulation of metabotropic glutamate receptors (mGluRs) in other brain regions (Glaum and Miller 1993; Nathanson et al. 1995), and the mGluR agonist tACPD has been reported to produce long-lasting potentiation in the hippocampus (Otani and Ben-Ari 1991; Radpour and Thomson 1992; Bortolotto et al. 1994), suggesting the possibility that NO and $\mathrm{CO}$ could be activated in parallel by stimulation of NMDA and mGluRs, respectively. We tested that possibility by examining the effect of inhibitors of heme oxygenase on potentiation by tACPD.

Consistent with previous reports, we found that, when paired with weak tetanic stimulation of the presynaptic fibers $(50 \mathrm{~Hz}, 0.5 \mathrm{sec})$, a short bath application of tACPD $(20 \mu \mathrm{M}, 2 \mathrm{~min})$ produced a rapid enhancement of the EPSP that lasted more than $1 \mathrm{hr}$ (Fig. 3A). Neither weak stimulation nor tACPD alone produced enhancement (Fig. 3A,B). Pretreatment with ZnPP (10 $\mu \mathrm{M})$ or ZnBG (10 $\mu \mathrm{M})$ completely abolished the long-lasting enhancement produced by tACPD paired with weak tetanic stimulation (Fig. 3C). As controls for specificity, ZnPP or ZnBG did not affect the baseline EPSP in a second, untetanized pathway in the same slice or post-tetanic potentiation (PTP) in the tetanized pathway. ZnPP or ZnBG also did not affect the decrementing potentiation 5 min after the weak tetanus (short-term potentiation or STP), suggesting that they did not affect NMDA receptors (Stevens and Wang 1993; Zhuo et al. 1993). Furthermore, the inactive analog CuPP did not affect the long-lasting enhancement produced by tACPD paired with weak stimulation (data not shown). Unlike ZnPP and ZnBG, the NO synthase inhibitor $N^{\omega}$ nitro-arginine $(100 \mu \mathrm{M})$ also did not affect the enhancement by tACPD paired with weak tetanic stimulation (Fig. 4A). These results further support the idea that the heme oxygenase inhibitors did not act by inhibiting NO synthase in these studies, and suggest that $\mathrm{CO}$ is involved in potentiation by tACPD.

If $\mathrm{CO}$ serves as one of the retrograde messengers during potentiation, it must diffuse from the postsynaptic cell to the presynaptic terminals through the extracellular space. Following bath application of the $\mathrm{CO}$ and NO binding protein hemoglobin $(20 \mu \mathrm{M})$ for at least $0.5 \mathrm{hr}$, tACPD paired

$$
\begin{array}{llllllllllllllll}
L & E & A & R & N & I & N & G & \boldsymbol{\bigotimes} \\
\mathbf{6 8} & & M & E & M & O & R & Y
\end{array}
$$



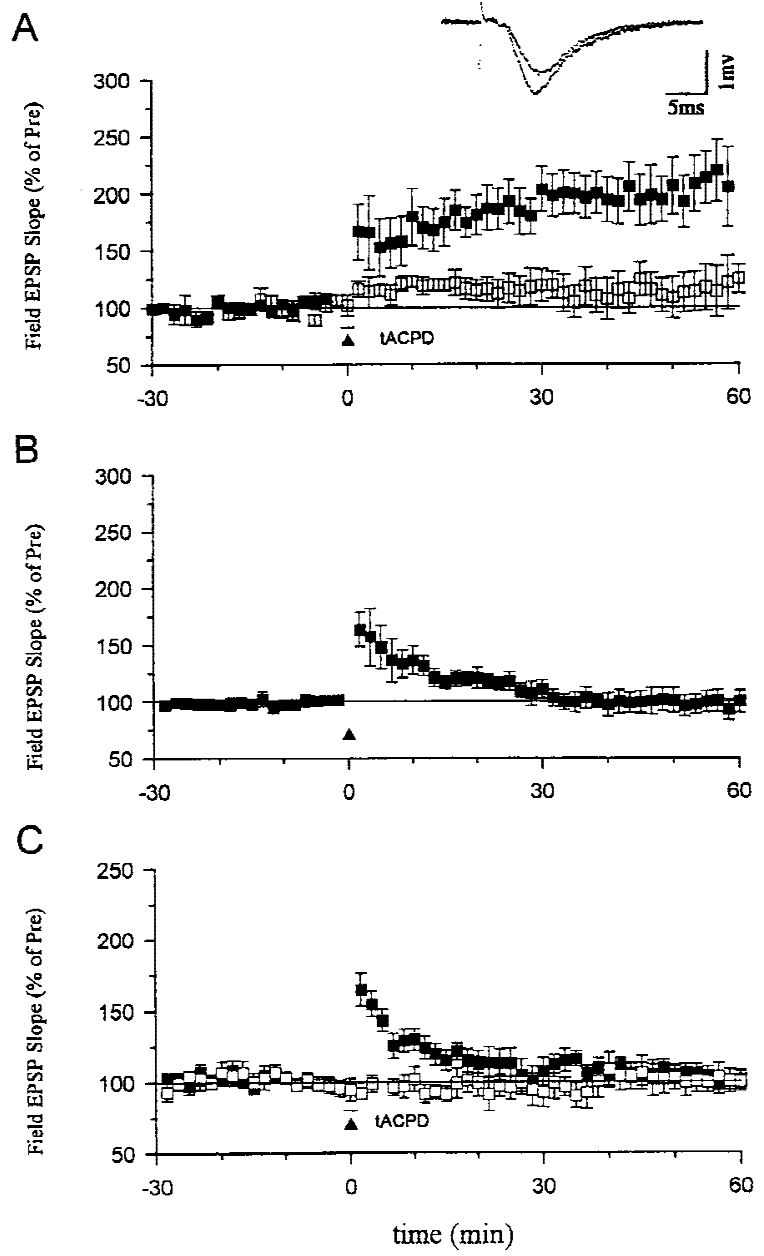

Figure 3: Effects of heme oxygenase inhibitors on longterm enhancement produced by tACPD. (A) tACPD (20 $\mu \mathrm{M}$, horizontal bar) produced a rapid onset, long-lasting enhancement of the field EPSP $(201.5 \pm 22.4 \%, n=8$, $t=4.53, P<0.01$, comparing average EPSPs 50-60 min post-training and $30 \mathrm{~min}$ pretraining) when applied at the same time as weak tetanic stimulation of the presynaptic fibers $(50 \mathrm{~Hz}, 0.5 \mathrm{sec}, \mathbf{\Delta}$ ) (paired training). The EPSP at a control pathway in the same slice, which received tACPD alone, was not significantly potentiated $(111.7 \pm 14.8 \%)$. ( $\square)$ tACPD alone; $(\mathbf{\square})$ tACPD paired. (Inset) Representative recordings of the field EPSP before and 60 min after tACPD paired training. (B) Weak stimulation $(50 \mathrm{~Hz}, 0.5 \mathrm{sec})$ alone did not produce long-term enhancement $(96.5 \pm 6.7 \%, n=7)$. (C) Heme oxygenase inhibitors (10 $\mu \mathrm{M}$ ZnPP-IX or ZnBG) completely blocked long-term enhancement produced by tACPD paired training $(\mathbb{\square})(\mathrm{ZnPP}-\mathrm{IX}, 113.9 \pm 8.2 \%, n=6$; ZnBG, $89.8 \pm 5.9 \%, n=5)$. The EPSP at a second control pathway in the same slice was stable ( $\square$, tACPD alone) $(100.7 \pm 7.6 \%)$. Results with the two inhibitors were similar and have been pooled. The average prevalues were $0.26 \mathrm{mV} / \mathrm{msec}$ (tACPD paired), 0.27 (tACPD alone) $(A)$; $0.26(B)$; and 0.25 (paired), 0.30 (control) $(C)$. with weak tetanic stimulation failed to produce long-lasting potentiation, but rather produced slight depression (Fig. 4B). This depression seems unlikely to result from the nonspecific effects of hemoglobin, because the EPSP in a second (control) pathway in the same slice was stable. One possible explanation is that tACPD might also trigger other processes related to depression that do not require diffusion of $\mathrm{CO}$ or NO through the extracellular space (Baskys and Malenka 1991; Bolshakov and Siegelbaum 1994).
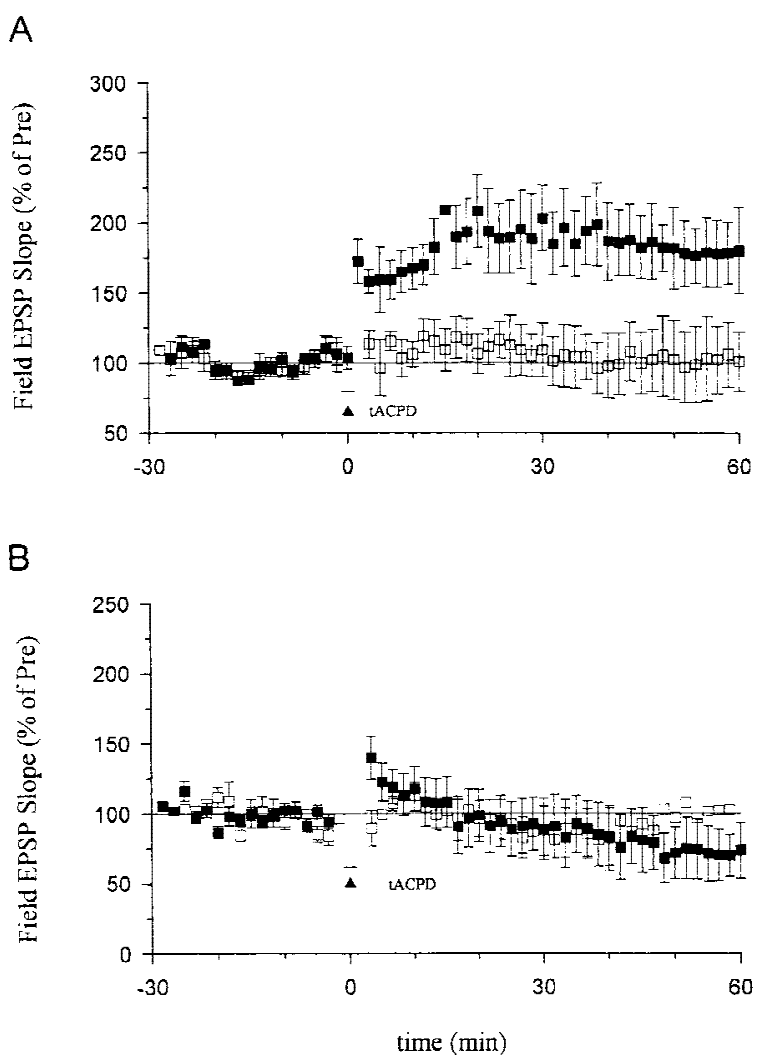

Figure 4: Effects of an NO synthase inhibitor or hemoglobin on long-term enhancement produced by tACPD paired training. $(A) N^{\omega}$-nitro-arginine $(100 \mu \mathrm{M})$ did not block long-term enhancement by tACPD paired with weak stimulation $(\boldsymbol{\square})(172.4 \pm 19.3 \%, n=8, t=3.75$, $P<0.01)$. The EPSP at a second control pathway in the same slice, which received tACPD alone, was stable $(\square)$ $(101.1 \pm 24.5 \%)$. (B) Hemoglobin $(20 \mu \mathrm{M})$ completely prevented long-term enhancement produced by tACPD paired training ( $\mathbf{\square})(72.0 \pm 19.5 \%, n=7)$. The EPSP at a second control pathway in the same slice was stable $(\square)$ $(104.3 \pm 36 \%)$. The average prevalues were $0.30 \mathrm{mV} /$ msec (paired), 0.26 (control) $(A)$ and 0.28 (paired), 0.30 (control) (B).

$$
\begin{array}{llllllllllllllll}
L & E & A & R & N & I & N & G & \begin{array}{l}
\boldsymbol{Q} \\
69
\end{array} & M & E & M & O & R & Y
\end{array}
$$




\section{INVOLVEMENT OF SOLUBLE GUANYLYL CYCLASE IN TACPD-INDUCED ENHANCEMENT}

CO activates soluble guanylyl cyclase and increases the production of CGMP in various regions of the central nervous system, including the hippocampus (J.T. Laitinen, K.S.M. Laitinen, L. Tuomisto, and M.M. Airaksinenn, unpubl.; Maine 1993; Verma et al. 1993). LY83583 and ODQ, two inhibitors of guanylyl cyclase, can block the induction of LTP (Zhuo et al. 1994; Boulton et al. 1995; Son et al. 1998). Conversely, membrane-permeable cGMP analogs paired with weak tetanic stimulation can produce long-lasting potentiation both in hippocampal slices (Haley et al. 1992; Zhuo et al. 1994; Son et al. 1998) and in dissociated cultures of hip-

A

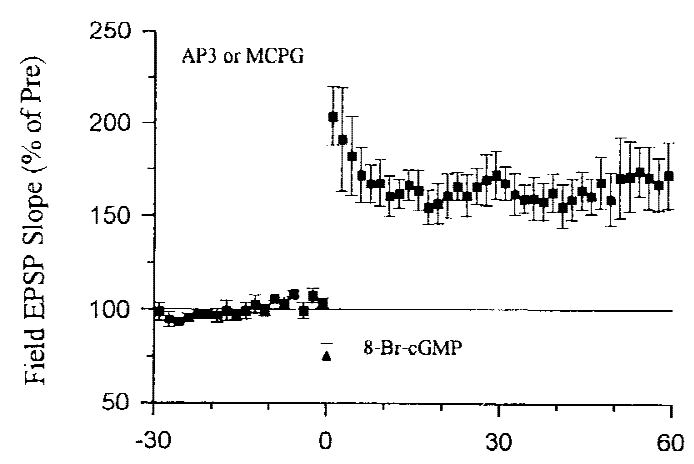

C

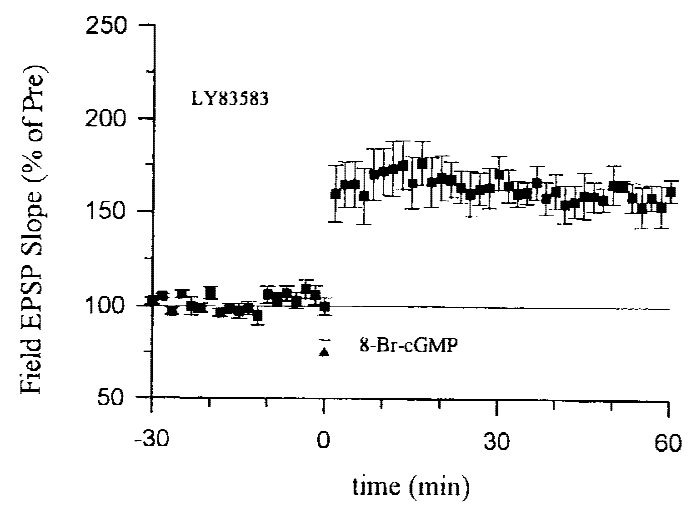

pocampal neurons (Arancio et al. 1995). Therefore, we investigated the relationship between mGluRs and cGMP during potentiation.

8-Br-cGMP paired with weak tetanic stimulation still produced significant long-term enhancement in the presence of the mGluR antagonists 2-amino-3-phosphonopropinate (AP3, $100 \mu \mathrm{M}$ ) or (+)-MCPG (500 $\mu \mathrm{M})$ (Fig. 5A), suggesting that cGMP does not act by enhancing activation of some types of mGluRs. Conversely, pretreatment with the guanylyl cyclase inhibitor LY83583 (5 $\mu \mathrm{M}$ ) abolished the enhancement produced by tACPD paired with weak tetanic stimulation (Fig. 5B), suggesting that cGMP acts downstream of the mGluRs. LY83583 did not affect the enhancement produced by $8-\mathrm{Br}-$ cGMP paired with weak tetanic stimulation (Fig.

B

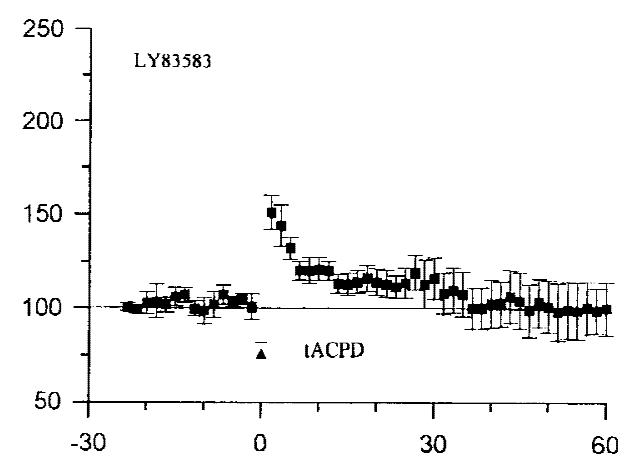

D

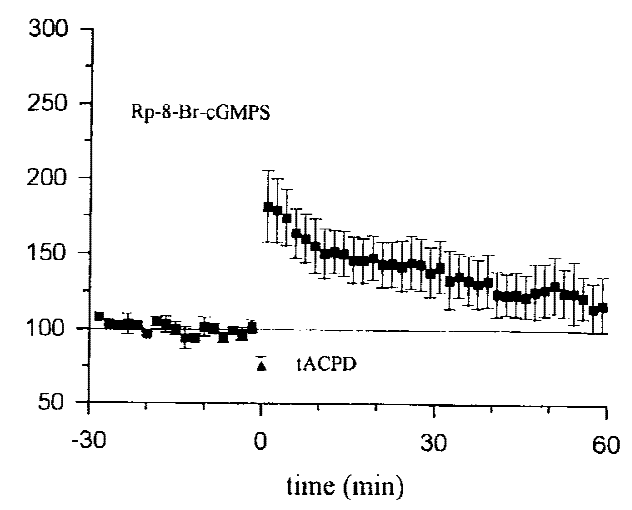

Figure 5: CGMP and cGMP-dependent protein kinase may act downstream of the mGluRs. $(A)$ The enhancement by 8-Br-cGMP (100 $\mu \mathrm{M}$, horizontal bar) paired with weak tetanic stimulation $(\mathbf{\Lambda})$ was not blocked by the mGluR antagonists AP3 $(181.5 \pm 14.9 \%, n=5, t=5.49, P<0.01)$ or $(+)$-MCPG $(159.7 \pm 11.9 \%, n=5, t=5.02, P<0.01)$. Results with the two antagonists were similar and have been pooled. $(B)$ The enhancement produced by tACPD $(20 \mu \mathrm{M})$ paired with weak tetanus was abolished by the guanylyl cyclase inhibitor LY83583 $(100.1 \pm 14.2 \%, n=6)$. $(C)$ The enhancement produced by 8 -Br-cGMP $(100 \mu \mathrm{M})$ paired with weak tetanus was not blocked by LY83583 (160.0 $\pm 10.0 \%, n=6)$. (D) Rp-8-BrcGMPS, an inhibitor of cGMP-dependent protein kinase, reduced the enhancement produced by tACPD (20 $\mu \mathrm{M})$ paired with weak tetanus $(120.6 \pm 16.7 \%, n=6, t=2.50, P<0.05$ compared with tACPD paired training in ACSF; Fig. 3A). The average prevalues were $0.30 \mathrm{mV} / \mathrm{msec}(A), 0.32(B), 0.42(C)$, and $0.32(D)$.

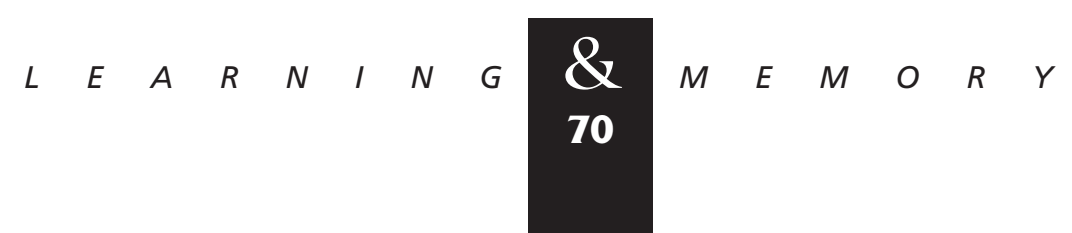


5C), indicating that LY83583 is relatively specific and did not inhibit processes downstream from soluble guanylyl cyclase. Furthermore, pretreatment with an inhibitor of cGMP-dependent protein kinase, Rp-8-Br-cGMPS (10 $\mu \mathrm{M})$ also significantly reduced the enhancement produced by tACPD paired with weak tetanic stimulation (Fig. 5D). These results suggest that mGluRs produce potentiation by stimulating guanylyl cyclase and cGMPdependent protein kinase.

As another test for activation of guanylyl cyclase by mGluRs, we carried out an in vitro biochemical assay of cGMP in hippocampal slices. Application of tACPD $(100 \mu \mathrm{M})$ produced an increase in cGMP (Fig. 6A). In agreement with previous reports (East and Garthwaite 1991; Chetkovich et al. 1993), NMDA or the NO donor sodium nitroprusside (SNP) also increased the level of cGMP in hippocampal slices.

\section{HEME OXYGENASE ACTIVITY IN HIPPOCAMPUS}

These results are consistent with the possibility that during tetanic stimulation, NO synthase and heme oxygenase are activated in parallel by stimulation of NMDA and mGluRs, respectively. However, another possibility is that heme oxygenase activity is constitutive and provides a tonic, background level of stimulation that is necessary for potentiation by either tetanic stimulation or tACPD. To attempt to distinguish between these possibilities, we estimated hippocampal heme oxygenase activity by measuring the conversion of $\left[{ }^{14} \mathrm{C}\right]$ heme to $\left[{ }^{14} \mathrm{C}\right]$ bilirubin by the concerted activity of heme oxygenase, NADPH-cytochrome $\mathrm{P}-450$ reductase and biliverdin reductase. In the CA1 region of the hippocampus, the mean basal heme oxygenase activity was $877 \pm 57$ pmoles $/ \mathrm{mg}$ per hour ( $n=25$ slices) when $21.4 \mu$ m substrate concentration was used. Pretreating slices with $\mathrm{ZnPP}(10 \mu \mathrm{M})$ for at least $30 \mathrm{~min}$ at $28^{\circ} \mathrm{C}$ significantly decreased heme oxygenase activity $\sim 40 \%$ (Fig. 6B). In contrast, $N^{\omega}$-nitro-arginine $(100 \mu \mathrm{M})$ did not affect heme oxygenase activity (data not shown). Strong tetanus or application of tACPD for either 2 or $5 \mathrm{~min}$ also did not affect heme oxygenase activity significantly.

The assay may not be sensitive enough to detect stimulation of heme oxygenase by tetanic stimulation if the tetanus releases endogenous substrates that compete with the exogenous heme in the assay. To test this possibility, we used a lower substrate concentration. However, the enzyme ac-

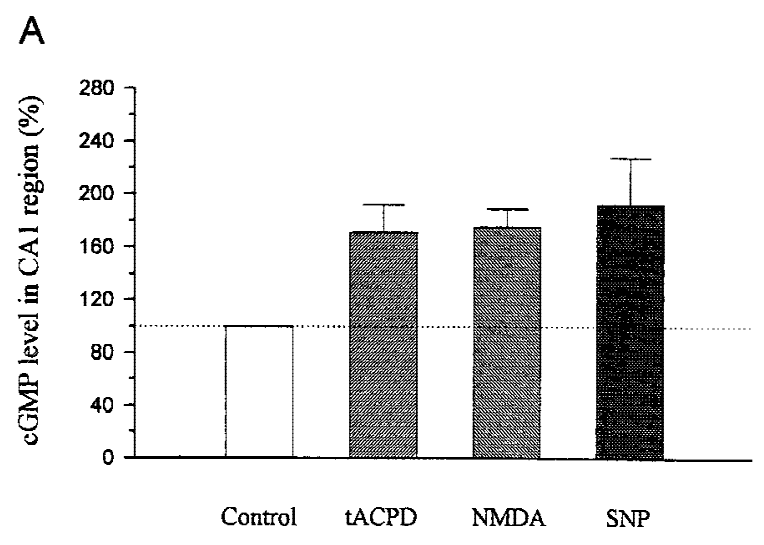

B

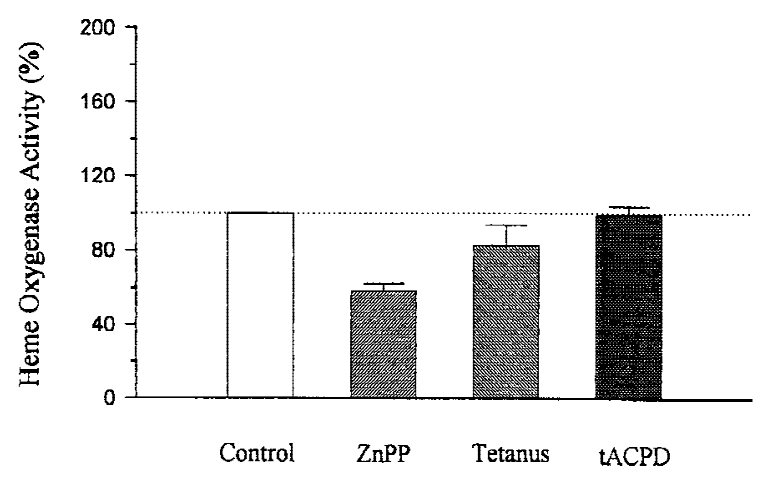

Figure 6: Biochemical assays. (A) Radioimmunoassay of CGMP in the CA1 region of hippocampus treated with vehicle (control), tACPD, NMDA, or SNP. The cGMP level was increased by $100 \mu \mathrm{m}$ tACPD $(170.8 \pm 20.9 \%, \quad n=6, \quad t=3.39, \quad P<0.05), \quad 10 \mu \mathrm{M}$ NMDA $(175.0 \pm 13.9 \%, n=3)$, or $300 \mu$ M SNP $(192.2 \pm 35.7 \%, n=3)$. Data are presented as the percentage of the cGMP level in vehicle-treated slices from the same experiment. (B) Heme oxygenase activity in slices treated with vehicle (control), ZnPP, strong tetanus, or tACPD. Pretreatment with ZnPP $(10 \mu \mathrm{M})$ for at least 30 min significantly decreased heme oxygenase activity to $58.3 \pm 3.8 \%$ of control values $(n=4$, $t=10.97, P<0.01)$. Strong tetanus did not affect heme oxygenase activity, measured $1 \mathrm{~min}$ after tetanic stimulation, with $21.4 \mu \mathrm{M}$ substrate concentration $(82.7 \pm 10.9 \%, n=4)$. Application of tACPD $(100 \mu \mathrm{M})$ for either 2 or $5 \mathrm{~min}$ did not significantly affect heme oxygenase activity (tACPD $2 \mathrm{~min}, 93.7 \pm 5.2 \%, n=5$; tACPD $5 \mathrm{~min}, 104.7 \pm 3.7 \%, n=5)$. The 2 - and 5 - $\min$ data have been pooled.

tivity was also not altered 5 min after strong tetanic stimulation with $4.4 \mu \mathrm{M}$ substrate concentration $(111.0 \pm 15.4 \%, n=6)$. Basal heme oxygenase activity was significantly lower $(227 \pm 54$ pmoles $/ \mathrm{mg}$ per hour, $n=8$ ) when assessed with $4.4 \mu \mathrm{m}$ substrate concentration, as compared with the activity

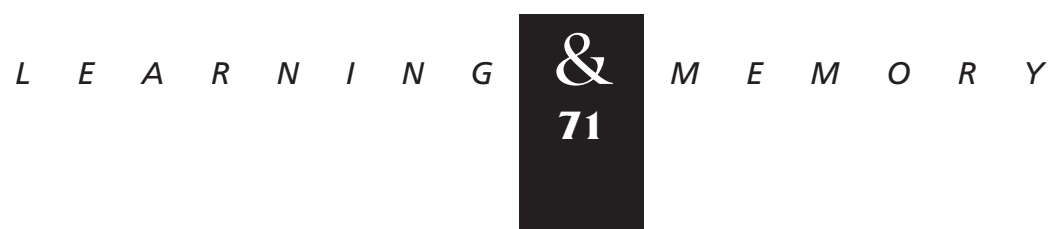


found with $21.4 \mu \mathrm{M}$ heme ( $t=6.09, P<0.001$ ), suggesting that substrate availability may be a ratelimiting step in hippocampal heme oxidation. This is somewhat surprising, as both known isozymes of heme oxygenase have a $K_{m} \sim 0.5 \mu \mathrm{M}$ for heme (Maines 1988). On the basis of a three-point Lineweaver-Burk plot, we estimated a $K_{m}$ of $37.2 \mu \mathrm{m}$ and a $v_{\max }$ of $2250 \mathrm{pmoles} / \mathrm{mg}$ per hour for the basal hippocampal heme-degrading capacity. This result may suggest the presence of additional heme-degrading capacity within the hippocampal homogenate. Heme-degrading capacity is not necessarily limited to the two known microsomal heme oxygenases, as there is some evidence for the presence of mitochondrial and cytosolic systems for heme degradation (Maines 1988). The possible implication of these findings is that the present studies may have underestimated the actual capacity of hippocampal tissue to generate CO.

The results of these assays favor the hypothesis that constitutive rather than stimulated heme oxygenase activity is critical for potentiation induced by strong tetanus or tACPD-paired training. However, our results do not exclude the possibility that heme oxygenase is activated in a restricted region or cellular compartment during LTP induction, and therefore might not be detected in our assays. Similarly, because we applied tetanic stimulation or tACPD to slices and then measured heme oxygenase activity in homogenates, we may not have detected rapidly reversible stimulation of heme oxygenase.

\section{Discussion}

LTP in CA1 and dentate gyrus can be blocked by inhibitors, targeted mutation, or adenovirus-mediated inhibition of NO synthase, suggesting that NO is involved in potentiation (Böhme et al. 1991; O'Dell et al. 1991; Schuman and Madison 1991; Haley et al. 1992; Mizutani et al. 1993; Boulton et al. 1995; Doyle et al. 1996; Kantor et al. 1996; Son et al. 1996; Wu et al. 1997). However, NO synthase inhibitors failed to block LTP in some studies (Kato and Zorumski 1993; Bannerman et al. 1994; Cummings et al. 1994), and in other studies NO synthase inhibitors blocked LTP only under some experimental circumstances, but not under other circumstances (Gribkoff and Lum-Ragan 1992; Chetkovich et al. 1993; Haley et al. 1993, 1996; Williams et al. 1993; O'Dell et al. 1994; Malen and Chapman 1997). Williams et al. (1993) suggested that NO synthase inhibitors block LTP only in slices prepared from young animals and maintained at room temperature. However, we and others have found that NO synthase inhibitors can block LTP in slices maintained at $28-32^{\circ} \mathrm{C}$ (Böhme et al. 1991; O'Dell et al. 1991, 1994; Gribkoff and Lum-Ragan 1992; Chetkovich et al. 1993; Haley et al. 1993; Boulton et al. 1995; Son et al. 1996; Malen and Chapman 1997; Wu et al. 1997) and also in vivo (Mizutani et al. 1993; Doyle et al. 1996). We did not examine LTP in older rats, but several studies (Doyle et al. 1996; Haley et al. 1996; Malen and Chapman 1997) have reported that NO synthase inhibitors can block LTP in older animals. Therefore, we feel that age and temperature cannot explain most of the differences in the published results, and suggest that more of those differences might be explained by differences in the tetanic stimulation. We found that an NO synthase inhibitor blocked LTP produced by one train of tetanic stimulation and significantly reduced LTP by two trains of stimulation, but only slightly reduced LTP produced by four trains of stimulation. Similar results have been obtained in several other studies in which the strength of the tetanic stimulation was varied in different ways (Chetkovich et al. 1993; Haley et al. 1993; O'Dell et al. 1994; Malen and Chapman 1997; but, see Gribkoff and Lum-Ragan 1992, for different results).

These results suggest that NO contributes importantly to LTP induced by relatively weak tetanic stimulation, but other messengers or enzymes contribute more to LTP induced by stronger tetanic stimulation. For example, previous studies have shown that cAMP-dependent protein kinase (PKA) makes little contribution to early-phase LTP induced by one train of tetanic stimulation, but makes more of a contribution to intermediatephase LTP induced by two trains and makes a large contribution to late-phase LTP induced by four trains (Huang and Kandel 1994; Blitzer et al. 1995; Winder et al. 1998). Consistent with these results, Lu et al. (in prep.) have recently found that cGMPdependent protein kinase (PKG) contributes to late-phase LTP induced by three trains, but makes less of a contribution to LTP induced by four trains. Thus, the NO-cGMP-PKG signaling pathway and the cAMP-PKA pathway appear to play complementary roles in LTP. In addition to these pathways, other messengers and enzymes most likely also make contributions to LTP that are larger or smaller depending on the experimental conditions.

Another candidate retrograde messenger is CO. Inhibitors of heme oxygenase also block or significantly reduce the induction of LTP in the

$$
\begin{array}{llllllllllllllll}
L & E & A & R & N & I & N & G & \begin{array}{l}
\boldsymbol{Z} \\
\mathbf{7 2}
\end{array} & M & E & M & O & R & Y
\end{array}
$$


hippocampus (Stevens and Wang 1993; Zhuo et al. 1993; Ikegaya et al. 1994; Poss et al. 1995). However, there are concerns about the specificity of heme oxygenase inhibitors, one being that they may also inhibit NO production (Meffert et al. 1994; Okada, 1996). This seems unlikely in the present study, because LTP induced by either four trains of tetanic stimulation or tACPD paired with weak tetanus was only slightly reduced by an inhibitor of NO synthase ( $N^{\omega}$-nitro-arginine) but was significantly reduced by two inhibitors of heme oxygenase (ZnBG and ZnPP) at a 10-fold lower dosage. This pattern of results might occur if $\mathrm{ZnBG}$ and ZnPP were actually better inhibitors of NO synthase than $N^{\omega}$-nitro-arginine. However, in biochemical assays of NO synthase activity in hippocampal tissue (East and Garthwaite 1991; Huang et al. 1993; Meffert et al. 1994) ZnBG did not inhibit NO synthase at all, and, although ZnPP did inhibit NO synthase, it was $\sim 1000$-fold less potent than $N^{\omega}$-nitro-arginine. Another concern is that inhibitors of heme oxygenase might also act by inhibiting soluble guanylyl cyclase (Ignarro et al. 1984; Luo and Vincent 1994). However, heme oxygenase inhibitors did not block potentiation induced by CO, which stimulates soluble guanylyl cyclase. These results are consistent with previous reports that heme oxygenase inhibitors do not inhibit soluble guanylyl cyclase in olfactory neuronal cultures (Verma et al. 1993), and that in endothelial and intestinal tissue heme oxygenase inhibitors are more selective for heme oxygenase than for either NO synthase or guanylyl cyclase (Zakhary et al. 1996, 1997). A third concern is that LTP appears to be normal in animals with a targeted mutation of heme oxygenase-2 (Poss et al. 1995). A possible explanation for this negative result is compensation by other isoforms of heme oxygenase or other messengers (Chen and Tonegawa 1996).

If both NO synthase and heme oxygenase are involved in LTP, it is not clear what their respective roles might be. One possibility suggested by our results is that they are activated by different stimulation patterns that might engage different receptors and second messengers. In many regions of the central nervous system, NO synthase activity is acutely controlled by glutamate NMDA receptors through a $\mathrm{Ca}^{2+}$-calmodulin-dependent mechanism. In the hippocampus, tetanic stimulation or NMDA application activates NO synthase, but the molecular mechanisms for activation of heme oxygenase are not known. Pharmacological studies with heme oxygenase inhibitors suggest that heme oxygenase may be activated in the brainstem or cerebellum by metabotropic glutamate receptor activation (Glaum and Miller 1993; Nathanson et al. 1995), suggesting the possibility that NO and CO could be activated in parallel by stimulation of NMDA and mGluRs, respectively. Consistent with this possibility, we found that inhibitors of heme oxygenase, but not NO synthase, block potentiation by the mGluR agonist tACPD.

Both NO and CO stimulate soluble guanylyl cyclase and increase the production of cGMP in hippocampus (East and Garthwaite 1991; Chetkovich et al. 1993; Verma et al. 1993). Furthermore, cGMP analogs can produce activity-dependent long-lasting potentiation and inhibitors of guanylyl cyclase or cGMP-dependent protein kinase can block LTP both in hippocampal slices (Haley et al. 1992; Zhuo et al. 1994; Boulton et al. 1995; Blitzer et al. 1995; Son et al. 1998) and in dissociated cultures of hippocampal neurons (Arancio et al. 1995). Other studies have failed to confirm some of these results (Schuman et al. 1994; Selig et al. 1996; Gage et al. 1997; Wu et al. 1998), but Son et al (1998) have recently identified experimental variables that may account in part for these discrepancies. We found that like LTP, tACPD-induced potentiation can be blocked by inhibitors of soluble guanylyl cyclase and cGMP-dependent protein kinase, and that tACPD stimulates guanylyl cyclase activity in hippocampal slices. These results suggest that mGluR activation might stimulate guanylyl cyclase through CO. However, in biochemical assays, we found that there is strong constitutive (basal) heme oxygenase activity in the CA1 region of hippocampus, and that this activity does not seem to be controlled by either tetanic stimulation or tACPD application. Although not conclusive, the assay results are most consistent with the possibility that heme oxygenase activity may not be acutely modulated by synaptic activation, as is NO synthase. Instead, constitutive heme oxygenase activity may be necessary for LTP and tACPDinduced potentiation, perhaps by producing tonic, background stimulation of guanylyl cyclase. If so, activation of mGluRs may produce acute stimulation of guanylyl cyclase through some other pathway, possibly arachidonic acid (Snider et al. 1984). Activation of mGluRs is thought to produce acute stimulation of guanylyl cyclase through NO in cerebellum (Okada 1995), but this seems unlikely in our experiments because potentiation by the mGluR agonist tACPD was blocked by inhibitors of guanylyl cyclase but not NO synthase.

$$
\begin{array}{llllllllllllllll}
\hline & E & A & R & N & I & N & G & \mathbf{Q} \\
\mathbf{7 3} & M & E & M & O & R & Y
\end{array}
$$


The most likely conclusion from our results, therefore, is that NO synthase and heme oxygenase are not activated in parallel during the induction of LTP, but rather that heme oxygenase plays a more tonic role. This idea is also more consistent with the results shown in Figures 1 and 2, which indicate that the NO synthase- and heme oxygenasedependent components of LTP are not additive. If heme oxygenase has a tonic function, it might act as a constitutive, housekeeping enzyme or it might have a more specific role as the source of a tonic retrograde messenger during LTP. Experiments on hippocampal neurons in culture support the idea that there may be both phasic and tonic retrograde messengers (J. Noel, A. Bergamaschi, and A. Malgaroli, unpubl.). One intriguing possibility is that although heme oxygenase may not play a phasic signaling role during the early phase of LTP, it could be induced by strong tetanic stimulation and thus play a more tonic role in the late, protein synthesis-dependent phase of LTP. Such a mechanism could contribute to a protein synthesis-dependent increase in presynaptic transmitter release during the late phase of LTP (Bolshakov et al. 1997; Sokolov et al. 1998).

\section{Acknowledgments}

We thank T. Abel, P. Nguyen, F. Rassendren, T. O'Dell, and S. Siegelbaum for their comments on an earlier draft, and A. Krawetz and $\mathrm{H}$. Ayers for typing the manuscript. This work was supported in part by grants from the National Institute on Aging (AG08702), the National Institute of Mental Health (MH50733), and the Howard Hughes Medical Institute.

The publication costs of this article were defrayed in part by payment of page charges. This article must therefore be hereby marked "advertisement" in accordance with 18 USC section 1734 solely to indicate this fact.

\section{References}

Arancio, O., E.R. Kandel, and R.D. Hawkins. 1995. Activity-dependent long-term enhancement of transmitter release by presynaptic $3^{\prime}, 5^{\prime}$-cyclic cGMP in cultured hippocampal neurons. Nature 376: 74-80.

Arancio, O., M. Kiebler., C.J. Lee, V. Lev-Ram, R.Y. Tsien, E.R. Kandel, and R.D. Hawkins. 1996. Nitric oxide acts directly in the presynaptic neuron to produce long-term potentiation in cultured hippocampal neurons. Cell 87: 1025-1035.

Bannerman, D.M., P.F. Chapman, P.A.T. Kelly, and R.G.M Morris. 1994. Inhibition of nitric oxide synthase does not prevent induction of long-term potentiation in vivo. J. Neurosci. 14: 7415-7425.

Baskys, A. and R.C. Malenka. 1991. Agonists at metabotropic glutamate receptors presynaptically inhibit EPSCs in neonatal rat hippocampus. J. Physiol. 444: 687-701.
Bliss, T.V.P. and G.L Collingridge. 1993. A synaptic model of memory: Long-term potentiation in the hippocampus. Nature 361: 31-39.

Blitzer, R.D., T. Wong, R. Nouranifar, R. lyengar, and E.M. Landau. 1995. Postsynaptic cAMP pathway gates early LTP in hippocampal CA1 region. Neuron 15: 1403-1414.

Böhme, G.A., C. Bon, J.M. Stutzmann, A. Doble, and J.C. Blanchard. 1991. Possible involvement of nitric oxide in long-term potentiation. Eur. J. Pharmacol. 199: 379-381.

Bolshakov, V.Y. and S.A. Siegelbaum. 1994. Postsynaptic induction and presynaptic expression of hippocampal long-term depression. Science 264: 1148-1152.

Bolshakov, V.Y., H. Golan, E.R. Kandel, and S.A. Siegelbaum. 1997. Recruitment of new sites of synaptic transmissin during the CAMP-dependent late phase of LTP at CA3-CA1 synapses in the hippocampus. Neuron 19: 635-651.

Bortolotto, Z.A., Z.I. Bashir, C.H. Davies, and G.L. Collingridge. 1994. A molecular switch activated by metabotropic glutamate receptors regulates induction of long-term potentiation. Nature 368: 740-743.

Boulton, C.L., E. Southam, and J. Garthwaite. 1995. Nitric-oxide dependent long-term potentiation is blocked by a specific inhibitor of soluble guanylyl cyclase. Neuroscience 69: 699-703.

Chen, C. and S. Tonegawa. 1996. Molecular genetic analysis of synaptic plasticity, activity-dependent neural development, learning, and memory in the mammalian brain. Annu. Rev. Neurosci. 20: 157-184.

Chetkovich, D.M., E. Klann, and J.D. Sweatt. 1993. Nitric oxide synthase-independent long-term potentiation in area CA1 of hippocampus. NeuroReport 4: 919-922.

Clark, G.D., L.T. Happel, C.F. Zorumski, and N.G. Bazan. 1992. Enhancement of hippocampal excitatory synaptic transmission by platelet-activating factor. Neuron 9: 1211-1216.

Cummings, J.A., S.M. Nicola, and R.C. Malenka. 1994. Induction in the rat hippocampus of long-term potentiation (LTP) and long-term depression (LTD) in the presence of a nitric oxide synthase inhibitor. Neurosci. Lett. 176: 110-114.

Del Cerro, S., A. Arai, and G. Lynch. 1990. Inhibition of long-term potentiation by an antagonist of platelet-activating factor receptors. Behav. Neurol. Biol. 54: 213-217.

Doyle, C., C. Holscher, M.J. Rowan, and R. Anwyl. 1996. The selective neuronal NO synthase inhibitor 7-nitro-indazole blocks both long-term potentiation and depotentiation of field EPSPs in rat hippocampal CA1 in vivo. J. Neurosci. 16: 418-424.

East, S.J. and J. Garthwaite. 1991. NMDA receptor activation in rat hippocampus induces cyclic GMP formation through

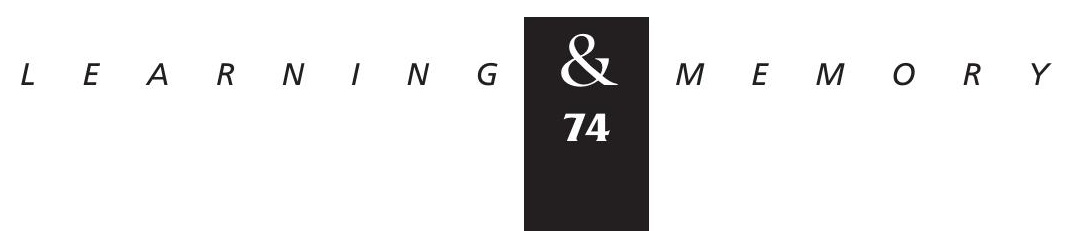


the L-arginine nitric oxide pathway. Neurosci. Lett. 123: $17-19$.

Gage, A.T., M. Reyes, and P.K. Stanton. 1997. Nitric-oxide-guanylyl-cyclase-dependent and independent components of multiple forms of long-term synaptic depression. Hippocampus 7: 288-295.

Glaum, S.R. and R.J. Miller. 1993. Zinc protoporphyrin-IX blocks the effects of metabotropic glutamate receptor activation in the rat nucleus tractus solitarii. Mol. Pharmacol. 43: 965-969.

Gribkoff, V.K. and J.T. Lum-Ragan. 1992. Evidence for nitric oxide synthase inhibitor-sensitive and insensitive hippocampal synaptic potentiation. J. Neurophysiol. 68: 639-642.

Haley, J. E., G.L. Wilcox, and P.F. Chapman. 1992. The role of nitric oxide in hippocampal long-term potentiation. Neuron 8: 211-216.

Haley, J. E., P.L. Malen, and P.F. Chapman. 1993. Nitric oxide synthase inhibitors block long-term potentiation induced by weak but not strong tetanic stimulation at physiological brain temperatures in rat hippocampal slices. Neurosci. Lett. 160: 85-88.

Haley, J., E. Schaible, P. Paulidis, A. Murdock, and D.V. Madison. 1996. Basal and apical synapses of CA1 pyramidal cells employ different LTP induction mechanisms. Learn. \& Mem. 3: 289-295.

Hawkins, R.D., E.R. Kandel, and S.A. Siegelbaum. 1993. Learning to modulate transmitter release: Themes and variations in synaptic plasticity. Annu. Rev. Neurosci. 16: 625-665.

Huang, P.L., T.M. Dawson, D.S. Bredt, S.H. Snyder, and M.C. Fishman. 1993. Targeted disruption of the neuronal nitric oxide synthase gene. Cell 75: 1273-1286.

Huang, Y.-Y. and E.R. Kandel. 1994. Recruitment of long-lasting and protein kinase A-dependent long-term potentiation in the CA1 region of hippocampus requires repeated tetanization. Learn. \& Mem. 1: 74-82.

Ignarro, L.J., B. Ballot, and K.S. Wood. 1984. Regulation of soluble guanylate cyclase activity by porphyrins and metalloporphyrins. J. Biol. Chem. 259: 6201-6207.

Ikegaya, Y., H. Saito, and N. Matsuki. 1994. Involvement of carbon monoxide in long-term potentiation in the dentate gyrus of anesthetized rats. Jpn. J. Pharmacol. 64: 225-227.

Kang, H. and E.M. Schuman. 1995. Long-lasting neurotrophin-induced enhancement of synaptic transmission in the adult hippocampus. Science 267: 1658-1662.

Kantor, D.B., M. Lanzrein, S.J. Stary, G.M. Sandoval, W.B. Smith, B.M. Sullivan, N. Davidson, and E.M. Schuman. 1996. A role for endothelial NO synthase in LTP revealed by adenovirus-mediated inhibition and rescue. Science 274: 1744-1748.

Kato, K. and C.F. Zorumski. 1993. Nitric oxide inhibitors facilitate the induction of hippocampal long-term potentiation by modulating NMDA responses. J. Neurophysiol. 70: 1260-1263.

Korte, M., O. Griesbeck, C. Grand, P. Carroll, V. Staiger, H. Thoenen, and T.Bonhoffer. 1996. Virus-mediated gene transfer into hippocampal CA1 region restores long-term potentiation in brain-derived neurotrophic factor mutant mice. Proc. Natl. Acad. Sci. 93: 12547-12552.

Laitinen, J.T. and R.O. Juronen. 1995. A sensitive microassay reveals marked regional differences in the capacity of rat brain to generate carbon monoxide. Brain Res.

694: 246-252.

Linden, D. J., K. Narasimban, and D. Gurfel. 1993.

Protoprophyrins modulate voltage-gated Ca current in AtT-20 pituitary cells. J. Neurophysiol. 70: 2673-2677.

Luo, D. and S.R. Vincent. 1994. Metalloporphyrins inhibit nitric oxide-dependent cGMP formation in vivo. Eur. J. Pharmacol. 267: 263-267.

Maines, M.D. 1988. Heme oxygenase: Function, multiplicity, regulatory mechanisms, and clinical applications. FASEB J. 2: 2557-2568.

1993. Carbon monoxide: An emerging regulator of cGMP in the brain. Mol. Cell. Neurosci. 4: 389-397.

Malen, P. and P.F. Chapman. 1997. Nitric oxide facilitates long-term potentiaton, but not long-term depression. J. Neurosci. 17: 2645-2651.

Martin, W., G.M. Villani, D. Jothianandan, and R.F. Furchgott. 1985. Selective blockade of endothelium-dependent and glycerol trinitrate-induced relaxation by hemoglobin and by methylene blue in the rabbit aorta. J. Pharmacol. Exp. Ther. 232: 708-716.

Meffert, M.K., J.E. Haley, E.M. Schuman, H. Schulman, and D.V. Madison. 1994. Inhibition of hippocampal heme oxygenase, nitric oxide synthase, and long-term potentiation by metalloporphyrins. Neuron 13: 1225-1233.

Mizutani, A., H. Saito, and K. Abe. 1993. Involvement of nitric oxide in long-term potentiation in the dentate gyrus in vivo. Brain Res. 605: 309-311.

Nathanson, J.A., C. Scavone, C. Scanlon, and M. McKee. 1995. The cellular $\mathrm{Na}^{+}$pump as a site of action for carbon monoxide and glutamate: A mechanism for long-term modulation of cellular activity. Neuron 14: 781-794.

O'Dell, T.J., R.D. Hawkins, E.R. Kandel, and O. Arancio. 1991. Tests of the roles of two diffusible substances in long-term potentiation: Evidence for nitric oxide as a possible early retrograde messenger. Proc. Natl. Acad. Sci. 88: 11285-11289.

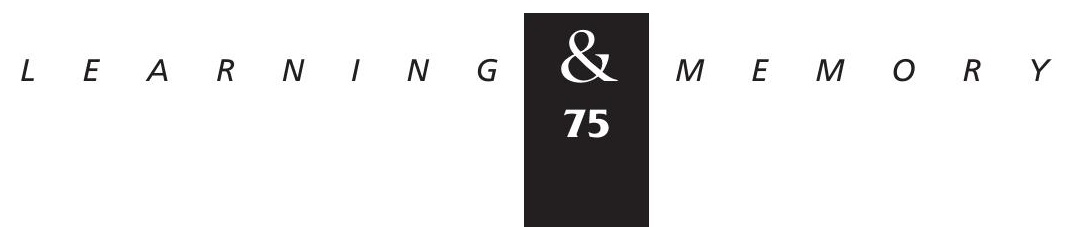


O'Dell, T.J., P.L. Huang, T.M. Dawson, J.L. Dinerman, S.H. Snyder, E.R. Kandel, and M.C. Fishman. 1994. Endothelial NOS and blockade of LTP by NOS inhibitors in mice lacking neuronal NOS. Science 265: 542-546.

Okada, D. 1995. Protein kinase C modulates calcium sensitivity of nitric oxide synthase in cerebellar slices. J. Neurochem. 64: 1298-1304.

1996. Zinc protoporphyrin IX suppresses nitric oxide production through a loss of L-arginine in rat cerebellar slices. Neurosci. Res. 25: 353-358.

Otani, S. and Y. Ben-Ari. 1991. Metabotropic receptor-mediated long-term potentiation in rat hippocampal slices. Eur. J. Pharmacol. 205: 325-326.

Poss, K.D., M.J. Thomas, A.K. Ebralidze, T.J. O'Dell, and S. Tonegawa. 1995. Hippocampal long-term potentiation is normal in heme oxygenase-2 mutant mice. Neuron 15: $867-873$.

Radpour, S. and A.M. Thomson. 1992. Synaptic enhancement induced by NMDA and Qp receptors and presynaptic activity. Neurosci. Lett. 138: 119-122.

Schuman, E.M. and D.V. Madison. 1991. A requirement for the intercellular messenger nitric oxide in long-term potentiation. Science 254: 1503-1506.

Schuman, E.M., M.K. Meffert, H. Schulman, and D.V. Madison. 1994. An ADP-ribosyl transferase as a potential target for nitric oxide action in hippocampal long-term potentiation. Proc. Natl. Acad. Sci. 91: 11958-11962.

Selig, D.K., M.R. Segal, D. Liao, R.C. Malenka, R. Malinow, R.A. Nicoll, and J.E. Lisman. 1996. Examination of the role of cGMP in long-term potentiation in the CA1 region of the hippocampus. Learn. \& Mem. 3: 42-48.

Snider, R.M., M. McKinney, C. Forray, and E. Richelson. 1984. Neurotransmitter receptors mediate cyclic GMP formation by involvement of arachidonic acid and lipoxygenase. Proc. Natl. Acad. Sci. 81: 3905-3909.

Sokolov, M.V., A.V. Rossokhin, T. Behnisch, K.G. Reymann, and L.L. Voronin. 1998. Interaction between paired-pulse facilitation and long-term potentiation of minimal excitatory postsynaptic potentials in rat hippocampal slices: $\mathrm{A}$ patch-clamp study. Neuroscience 85: 1-13.

Son, H., R.D. Hawkins, K. Martin, M. Kiebler, P.L. Huang, M.C. Fishman, and E.R. Kandel. 1996. Long-term potentiation is reduced in mice that are doubly mutant in endothelial and neuronal nitric oxide synthase. Cell 87: 1015-1023.

Son, H., Y.-F. Lu, M. Zhuo, O. Arancio, E.R. Kandel, and R.D. Hawkins. 1998. The specific role of cGMP in hippocampal LTP. Learn. \& Mem. 5: 231-245.

Stevens, C.F. and Y.-Y. Wang. 1993. Reversal of long-term potentiation by inhibitors of heme oxygenase. Nature 364: 147-149.
Thoenen, H. 1995. Neurotrophins and neuronal plasticity. Science 270: 593-598.

Verma, A., D.J. Hirsch, C.F. Glatt, G.V. Ronnett, and S.H. Snyder. 1993. Carbon monoxide: A putative neural messenger. Science 259: 381-384.

Wieraszko, A., G. Li, E. Kornecki, M.V. Hogan, and Y.H. Ehrlich. 1993. Long-term potentiation in the hippocampus by platelet-activating factor. Neuron 10: 553-557.

Williams, J.H., M.L. Errington, M.A. Lynch, and T.V.P. Bliss. 1989. Arachidonic acid induces a long-term activity-dependent enhancement of synaptic transmission in the hippocampus. Nature 341: 739-742.

Williams, J.H., Y.G. Li, A. Nayak, M.L. Errington, K.-P.S.J. Murphy, and T.V.P. Bliss. 1993. The suppression of long-term potentiation in rat hippocampus by inhibitors of nitric oxide synthase is temperature- and age-dependent. Neuron 11: $877-884$.

Winder, D.G., I.M. Mansuy, M. Osman, T.M. Moallem, and E.R. Kandel. 1998. Genetic and pharmacological evidence for a novel, intermediate phase of long-term potentiation suppressed by calcineurin. Cell 92: 25-37.

Wu, J., Y. Wang, M.J. Rowan, and R. Anwyl. 1997. Evidence for the involvement of the neuronal isoform of nitric oxide synthase during induction of long-term potentiation and long-term depression in the rat dentate gyrus in vitro. Neuroscience 78: 393-398.

1998. Evidence for involvement of the cGMP-protein kinase $G$ signaling system in the induction of long-term depression, but not long-term potentiation, in the dentate gyrus in vitro. J. Neurosci. 18: 3589-3596.

Zakhary, R., S.P. Gaine, J.L. Dinerman, M. Ruat, N.A. Flavahan, and S.H. Snyder. 1996. Heme oxygenase 2: Endothelial and neuronal localization and role in endothelium-dependent relaxation. Proc. Natl. Acad. Sci. 93: 795-798.

Zakhary, R., K.D. Poss, S.R. Jaffrey, C.D. Ferris, S. Tonegawa, and S. Snyder. 1997. Targeted gene deletion of heme oxygenase 2 reveals neural role for carbon dioxide. Proc. Natl. Acad. Sci. 94: 14848-14853.

Zhuo, M., S.A. Small, E.R. Kandel, and R.D. Hawkins. 1993. Nitric oxide and carbon monoxide produce activity-dependent long-term synaptic enhancement in hippocampus. Science 260: 1946-1950.

Zhuo, M., Y. Hu, C. Schultz, E.R. Kandel, and R.D. Hawkins. 1994. Role of guanylyl cyclase and cGMP-dependent protein kinase in long-term potentiation. Nature 368: 635-639.

Received June 26, 1998; accepted in revised form October 16,1998

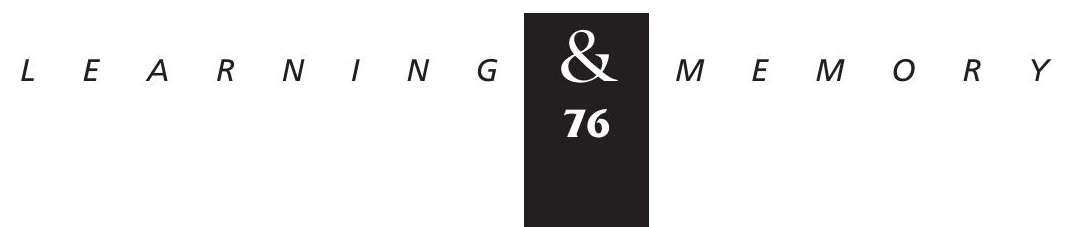




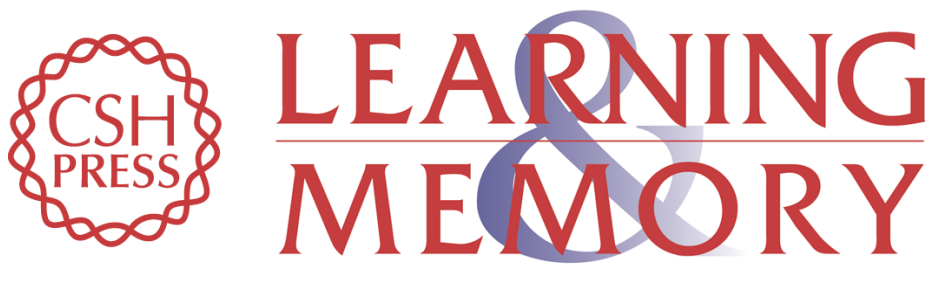

\section{Conditioning Method Dramatically Alters the Role of Amygdala in Taste Aversion Learning}

Glenn E. Schafe, Todd E. Thiele and llene L. Bernstein

Learn. Mem. 1998, 5:

Access the most recent version at doi:10.1101//m.5.6.481

\section{Related Content Errata for vol. 5, p. 481}

Learn. Mem. January, 1999 6: 62

References This article cites 29 articles, 4 of which can be accessed free at: http://learnmem.cshlp.org/content/5/6/481.full.html\#ref-list-1

Articles cited in:

http://learnmem.cshlp.org/content/5/6/481.full.html\#related-urls

License

Email Alerting Receive free email alerts when new articles cite this article - sign up in the box at the Service top right corner of the article or click here. 